\title{
Seleção de modelos polinomiais para representar o perfil e volume do fuste de Tectona grandis L.f.
}

\author{
Evandro Orfanó FIGUEIREDO ${ }^{1}$, José Roberto Soares SCOLFORO², Antônio Donizette de OLIVEIRA²
}

\begin{abstract}
RESUMO
A precisão do volume de um povoamento florestal torna-se importante à medida que as empresas florestais integram verticalmente suas atividades e o resíduo da elaboração de um produto torna-se matéria-prima para outros. Os estudos realizados objetivaram avaliar a acurácia dos modelos polinomiais propostos por Schöepfer (1966), Hradetzky (1976) e Goulding \& Murray (1976), na estimativa dos diâmetros e volumes ao longo do fuste de Tectona grandis L.f. de quatro povoamentos localizados na microrregião do Baixo Rio Acre e, ainda, testar a identidade do melhor modelo polinomial, avaliando-se a adequação de manter as áreas agrupadas ou segregálas em grupos menores ou individualmente. A base de dados foi constituída de 159 árvores cubadas rigorosamente. Na avaliação da acurácia dos modelos foram empregadas estatísticas de desvio médio, desvio padrão das diferenças, soma dos quadrados dos resíduos relativos e resíduos percentuais. O modelo Goulding \& Murray (1976) gerou as melhores estimativas de diâmetros e volumes ao longo do fuste, seguido pelos modelos Hradetzky (1976) e Schöepfer (1966). O teste de identidade de modelo mostrou ser mais adequado realizar ajustes independentes para as áreas $1 \mathrm{e} 4 \mathrm{e}$ para o subgrupo $2 \mathrm{e} 3$.
\end{abstract}

PALAVRAS-CHAVE

Volumetria, função de afilamento, taper, identidade de modelos, teca, estimativas de diâmetros e volumes.

\section{Selection of polynomial models to represent the profile and volume of the bole of Tectona grandis $L . f$.}

\begin{abstract}
The precise estimate of the volume of a forest stand become important as forest enterprises integrate vertically their activities and the residue of the manufacture of a product becomes the raw material for the manufacture of other. The accomplished studies aimed to evaluate the accuracy of the polynomial models proposed by Schöepfer (1966), Hradetzky (1976) and Goulding \& Murray (1976) in the estimate of the diameters and volumes along the bole of Tectona grandis L.f from four stands situated in the Baixo Rio Acre micro region and further, to test the identity of the best polynomial model by evaluating the adequacy of maintaining the grouped areas or segregate them into smaller groups or singly. The data base was made up of 159 trees scaled rigorously. In the evaluation of the accuracy of the models, the statistics of average deviation, standard deviation of differences, square summation of relative residue and percent residue. The Goulding \& Murray (1976) model was generated the best estimates of diameters and volume along the bole, followed by the models of Hradetzky (1976) and Schöepfer (1966). The model identity test proved more adequate to perform independent fit for areas 1 and 4 and for subgroup 2 and 3 .
\end{abstract}

\section{KEY WORDS}

Volumetry, tapering function, taper, identity of models, teak, diameter and volume estimates.

${ }^{1}$ Engenheiro Agrônomo, M.Sc., Pesquisador da Embrapa Acre, BR 364, km 14, Caixa Postal 321, CEP 69908-970, Rio Branco - AC; orfano@cpafac.embrapa.br. 2Professor do Departamento de Ciências Florestais da UFLA - Caixa Postal 37, Lavras - MG - CEP 37.200-000. scolforo@ufla.br; donizete@ufla.br 


\section{INTRODUÇÃO}

O planejamento e a otimização das atividades de exploração de florestas plantadas são influenciados pela precisão das estimativas volumétricas para uma determinada área. Dentre os vários métodos para viabilizar a estimativa dos volumes, pode-se citar as equaçōes de volume de simples entradas ou locais, as equações de volume de dupla entrada ou regionais, as equações de volume associadas à relação hipsométrica, os fatores de forma, as funções spline e as funções de afilamento. Esta última é representada por modelos matemáticos que representam o perfil da árvore e possibilitam a estimativa do diâmetro para qualquer altura ao longo do fuste ou vice-versa. Propicia assim, elementos para que se possa estimar o número de produtos madeireiros que a árvore pode produzir, assim como seus volumes.

Os estudos da acurácia de suas estimativas têm mostrado que estes modelos são uma poderosa ferramenta na administração de empreendimentos florestais, sem que haja ônus nos custos do inventário florestal. Entre as tendências de modelagem podemse citar: i. modelos simplificados: que não consideram a altura total, mas a distância entre um diâmetro qualquer (d) e a extremidade da árvore, além do diâmetro à altura do peito DAP; ii. modelos polinomiais: baseados principalmente na comparação entre a variável dependente $(\mathrm{d} / \mathrm{DAP})^{2}$ ou $\left(\mathrm{d} / \mathrm{D}_{0,1}\right)^{2}$ e os polinômios formados pela variável independente $(\mathrm{h} / \mathrm{H})$; iii. modelos sigmóides: utilizam transformaçôes de variáveis de modo que o modelo se assemelha à forma natural dos troncos. Trata-se de expressões matemáticas que geram um perfil semelhante a uma curva sigmóide; iv. modelos segmentados: consistem no uso de submodelos justapostos, cada um representando uma porção do tronco. A união de submodelos se dá por meio da imposiçāo de restrições ao modelo geral; v. modelos definidos por meio de análise multivariada: baseiam-se na aplicação de análise multivariada (análise de componentes principais) para investigar as variaçōes existentes na estrutura dos dados e definir o modelo de regressão a ser utilizado para descrever o perfil do tronco; e, vi. modelos trigonométricos.

Além das vantagens referentes à flexibilidade e amplitude das informaçōes geradas, as funções de afilamento apresentam ainda o princípio da compatibilidade entre o volume total e os volumes comerciais estimados. Tal princípio garante que a soma dos volumes comerciais seja sempre menor que o volume total (Scolforo, 1997).

Assim, este estudo teve como objetivos: (a) estudar e avaliar a acurácia dos modelos polinomiais de quinto grau, proposto por Schöepfer (1966); o modelo polinomial com potências inteiras e fracionárias, proposto por Hradetzky (1976); e, o modelo de polinomial de Goulding \& Murray (1976) para a estimativa dos diâmetros e volumes ao longo do fuste de Tectona grandis L.f., considerando a base de dados de quatro povoamentos; e, (b) testar a identidade do melhor modelo polinomial, avaliando- se a adequação de manter as áreas agrupadas ou segregá-las em grupos menores ou individualmente.

\section{MATERIAL E MÉTODOS}

\section{CARACTERIZAÇÃO DAS ÁREAS DE ESTUDO E DOS PLANTIOS ESTUDADOS}

Os estudos foram realizados em quatro povoamentos de teca plantados em três propriedades rurais, nos municípios de Rio Branco e Acrelândia, situados na microrregião do Baixo Rio Acre, no estado do Acre, Brasil.

A área 1 constitui-se num povoamento de teca com 9,52 anos de idade e densidade inicial de 2.083 árvores. ha $^{-1}$ (espaçamento de 2 × 2,4 metros), e a área 2 com 2,54 anos e densidade de 1.667 árvores.ha ${ }^{-1}$ (espaçamento de $2 \times 3$ metros).

A área 3, com 7,4 anos, foi implantada na densidade inicial de 1.111 árvores.ha ${ }^{-1}$ da espécie teca e 556 árvores. ha ${ }^{-1}$ da espécie mogno (Swietenia macrophylla King), porém, dois anos após o plantio, a população de mogno limitava-se a poucas árvores, em decorrência do forte ataque de broca (Hypsipyla grandella) e à intensa competição imposta pela teca. Atualmente, considera-se a área como um povoamento puro de teca com 1.111 árvores.ha${ }^{1}$, plantadas em filas duplas de $3 \times 2$ metros e com 4 metros entre fileiras duplas.

A Área 4, situada no município de Acrelândia, com 6,45 anos, foi instalada no espaçamento de $2 \times 3$ metros, perfazendo uma densidade inicial de 1.667 árvores.ha ${ }^{-1}$.

O material genético dos quatro povoamentos é da variedade Tennasserim, de procedência da Birmânia (Myanmar) para Trinidad.

\section{CUBAGEM RIGOROSA}

A amostra das árvores cubadas rigorosamente foi baseada na distribuição diamétrica dos povoamentos, cujas classes de diâmetro foram construídas com um diâmetro à altura do peito $\left(\mathrm{DAP}_{1,30 \mathrm{~m}}\right)$ mínimo de $5 \mathrm{~cm}$ e as amplitudes de classes delimitadas a cada 4 centímetros (Tabela 1 ).

Para cada árvore-amostra foram realizadas determinaçōes de altura total $\left(\mathrm{H}_{\mathrm{r}}\right)$, DAP e altura comercial até o diâmetro limite de $3 \mathrm{~cm}$ e duas medidas de espessura de casca em todas as posiçōes de diâmetro ao longo do fuste. Os diâmetros foram medidos nas alturas de $0,00 \mathrm{~m}, 0,25 \mathrm{~m}, 0,50 \mathrm{~m}, 0,75 \mathrm{~m}, 1,00 \mathrm{~m}, 1,30 \mathrm{~m}$, $1,50 \mathrm{~m}$ e, posteriormente, a cada $0,50 \mathrm{~m}$ até atingir a altura comercial. Com as tomadas de diâmetro nas citadas alturas absolutas, optou-se pelo método de Smalian para calcular os volumes, conforme descrito por Husch et al. (1982).

\section{MODELOS DE AFILAMENTOS TESTADOS}

O procedimento de ajuste foi realizado considerando-se os DAP de 1,3 m do solo com casca, para a base de dados das quatro áreas de estudo em conjunto. Posteriormente, as equaçōes ajustadas foram avaliadas pelo teste de identidade de modelos. 


\section{POLINÔMIO DE QUINTO GRAU (SCHÖEPFER, 1966).}

Este é um dos modelos de afilamento mais difundidos no meio florestal. O modelo é expresso por:

$$
\frac{d_{i}}{D A P}=\beta_{0}+\beta_{1}\left(\frac{h_{i}}{H_{t}}\right)+\beta_{2}\left(\frac{h_{i}}{H_{t}}\right)^{2}+\beta_{3}\left(\frac{h_{i}}{H_{t}}\right)^{3}+\beta_{4}\left(\frac{h_{i}}{H_{t}}\right)^{4}+\beta_{5}\left(\frac{h_{i}}{H_{t}}\right)^{5}+\varepsilon_{i}
$$

em que: $\beta_{\text {is }}=$ parâmetros a serem estimados; $d_{i}=$ diâmetro comercial $(\mathrm{cm})$ ou diâmetro correspondente a qualquer altura $h_{i}$, especificada ou comercial; DAP = diâmetro a 1,3 $\mathrm{m}$ de altura $(\mathrm{cm}) ; \mathrm{H}_{\mathrm{t}}=$ altura total $(\mathrm{m}) ; \mathrm{h}_{\mathrm{i}}=$ altura comercial $(\mathrm{m}) ; \mathrm{e}, \varepsilon_{\mathrm{i}}=$ erro de estimativa.

Para a obtenção dos diâmetros a diversas alturas ao longo do fuste, basta isolar (d), obtendo-se assim a função de afilamento, sendo:

$$
\frac{d_{i}}{D A P}=\beta_{0}+\beta_{1}\left(\frac{h_{i}}{H_{t}}\right)+\beta_{2}\left(\frac{h_{i}}{H_{t}}\right)^{2}+\beta_{3}\left(\frac{h_{i}}{H_{t}}\right)^{3}+\beta_{4}\left(\frac{h_{i}}{H_{t}}\right)^{4}+\beta_{5}\left(\frac{h_{i}}{H_{t}}\right)^{5}+\mathcal{E}_{i}
$$

Para se obter o volume $(\mathrm{V})$, é necessário integrar suas áreas seccionais $\left(\mathrm{g}_{\mathrm{i}}\right)$ entre o limite inferior $\left(\mathrm{h}_{1}\right)$ e superior $\left(\mathrm{h}_{2}\right)$ que se deseja. No caso de uma árvore, se o volume total é desejado, então $h_{1}=0$ e $h_{2}=$ altura total da árvore. A representação da integral é:

$$
V=K \int_{h_{1}}^{h_{2}} d_{i}^{2} \delta h
$$

em que: $\mathrm{K}=\pi / 40000 ; \mathrm{d}_{\mathrm{i}}=$ diâmetro correspondente a qualquer altura $h_{i}$ ao longo do fuste.

Resolvendo a integral da expressão (2), obtém-se:

$$
\begin{aligned}
\mathrm{V}= & \mathrm{K} \mathrm{DAP}{ }^{2}\left[\mathrm{c}_{0}^{2} h_{i}+\mathrm{c}_{1} \mathrm{c}_{0} h_{i}{ }^{2}+\left(2 / 3 \mathrm{c}_{2} \mathrm{c}_{0}+1 / 3 \mathrm{c}_{1}^{2}\right) h_{i}{ }^{3}+\left(1 / 2 \mathrm{c}_{3} \mathrm{c}_{0}+1 / 2 \mathrm{c}_{2} \mathrm{c}_{1}\right) h_{i}{ }^{4}\right. \\
+ & \left(2 / 5 \mathrm{c}_{4} \mathrm{c}_{0}+2 / 5 \mathrm{c}_{3} \mathrm{c}_{1}+1 / 5 \mathrm{c}_{2}^{2}\right) h_{i}{ }^{5}+\left(1 / 3 \mathrm{c}_{4} \mathrm{c}_{1}+1 / 3 \mathrm{c}_{5} \mathrm{c}_{0}+1 / 3 \mathrm{c}_{3} \mathrm{c}_{2}\right) h_{i}{ }^{6} \\
+ & \left(2 / 7 \mathrm{c}_{5} \mathrm{c}_{1}+2 / 7 \mathrm{c}_{4} \mathrm{c}_{2}+1 / 7 \mathrm{c}_{3}^{2}\right) h_{i}{ }^{7}+\left(1 / 4 \mathrm{c}_{5} \mathrm{c}_{2}+2 / 7 \mathrm{c}_{4} \mathrm{c}_{3}\right) h_{i}{ }^{8} \\
& \left.\left(2 / 9 \mathrm{c}_{5} \mathrm{c}_{3}+1 / 9 \mathrm{c}_{4}^{2}\right) h_{i}{ }^{9}+1 / 5 \mathrm{c}_{5} \mathrm{c}_{4} h_{i}{ }^{19}+1 / 11 \mathrm{c}_{5}{ }_{5} h_{i}{ }^{11}\right]_{h 2}^{h 1}+\varepsilon_{i}
\end{aligned}
$$

$\mathrm{K}, \mathrm{h}_{\mathrm{i},} \varepsilon_{\mathrm{i}}$ e DAP foram definidos anteriormente.

\section{POLINÔMIO DE POTÊNCIAS FRACIONÁRIAS E INTEIRAS} (HRADETZKY, 1976).

Segundo Ferreira (2004), o modelo Hradetzky (1976) diferencia-se do modelo proposto por Schöepfer (1966) por apresentar potências fracionárias e inteiras como tentativa de melhor representar o perfil da árvore. Schöepfer (1966) já relatava que o polinômio do $5^{\circ}$ grau, embora represente bem o perfil da árvore, não representa tão bem na porção inferior, já que há mais tortuosidade e sinuosidade na base da árvore, que nas partes superiores. Por isso, Hradetzky (1976) propôs potências mais apropriadas na descrição da forma da árvore, apontando como sugestão a construção do modelo pelo método Stepwise, para expoentes variando de 0,005 a 25 . Scolforo (2005) considera que Hradetzky (1976) sugeriu a utilização de potências inteiras da ordem de dezenas para representar o topo da árvore em conjunto com potências fracionárias para representar a porção inferior do fuste.

O procedimento de seleção das potências inteiras e fracionárias empregou o método estatístico Stepwise, cujo procedimento seleciona o conjunto de variáveis independentes que produza a melhor regressão (passo a passo), levando em consideração os valores mais elevados da estatística F (SAS, 1990).

Os expoentes testados foram: 0,$00005 ; 0,00001 ; 0,0009$; 0,$0007 ; 0,0006 ; 0,0004 ; 0,0002 ; 0,0001 ; 0,009 ; 0,008$; 0,$007 ; 0,006 ; 0,005 ; 0,004 ; 0,09 ; 0,08 ; 0,07 ; 0,06 ; 0,05$; 0,$04 ; 0,03 ; 0,02 ; 0,01 ; 0,9 ; 0,7 ; 0,6 ; 0,5 ; 0,4 ; 0,2 ; 0,1 ; 1 ; 2 ; 3$; $4 ; 5 ; 6 ; 7 ; 8 ; 9 ; 10 ; 15 ; 20 ; 25 ; 30 ; 35 ; 40 ; 45 ; 50 ; 55 ; 60 ; 65$; 70; 75; 80; 85; 90; e, 95 .

A forma de ajuste do polinômio é:

$$
\mathrm{d}_{\mathrm{i}}=\operatorname{DAP} \cdot\left[\beta_{0}+\beta_{1}\left(\frac{\mathrm{h}_{\mathrm{i}}}{\mathrm{H}_{\mathrm{t}}}\right)^{\mathrm{pl}}+\beta_{2}\left(\frac{\mathrm{h}_{\mathrm{i}}}{\mathrm{H}_{\mathrm{t}}}\right)^{\mathrm{p} 2}+\ldots+\beta_{\mathrm{n}}\left(\frac{\mathrm{h}_{\mathrm{i}}}{\mathrm{H}_{\mathrm{t}}}\right)^{\mathrm{pn}}\right]+\varepsilon_{\mathrm{i}}
$$

em que $d_{i}, D A P, h_{i}, H_{t}, b_{i}$, e $\beta_{i}$ foram definidos anteriormente.

Simplificando-se a expressão 4 por: $\mathrm{c}_{0}=\beta_{0}$ e $\mathrm{c}_{\mathrm{i}}=\left(\beta_{i} / \mathrm{H}_{\mathrm{t}}^{\mathrm{p}_{\mathrm{j}}}\right)$, em que $\mathrm{i}=1,2, \ldots, \mathrm{n} ; \mathrm{e}, \mathrm{p}_{\mathrm{i}}=$ expoentes selecionados pelo processo Stepwise, a expressão (4) assume a forma:

$$
d_{i}=D A P \cdot\left(C_{0}+c_{1} h_{1}^{p 1}+c_{2} h_{2}^{p 2}+\ldots+c_{n} h_{n}^{p n}\right)+\varepsilon_{i}
$$

Tabela 1 - Freqüência de árvores cubadas de Tectona grandis L.f. para os quatro povoamentos, nas diferentes classes de diâmetro.

\begin{tabular}{cccccccc}
\hline & & Área 1 & Área 2 & Área 3 & \multicolumn{2}{c}{ Área 4 } \\
\cline { 3 - 7 } & & \multicolumn{5}{c}{ Idade em anos } \\
\hline Número de classes & Classe de diâmetro $(\mathrm{cm})$ & 9,52 & 2,54 & 7,40 & 6,45 & Freqüência \\
\hline 1 & $5 \mid--9$ & 15 & 15 & 15 & 15 & 60 \\
2 & $9 \mid--13$ & 15 & - & 15 & 15 & 45 \\
3 & $13 \mid--17$ & 15 & - & 15 & 15 & 45 \\
4 & $17 \mid-21$ & 9 & - & - & 45 & 9 \\
\end{tabular}


O volume total ou de qualquer porção da árvore (sortimento) é obtido pela resolução da integral do polinômio (5), ficando a expressão da seguinte forma:

$$
\begin{aligned}
& \mathrm{V}=\mathrm{K} \cdot \mathrm{DAP} \mathrm{P}^{2} \cdot\left[\mathrm{c}_{0}^{2} \mathrm{~h}_{\mathrm{i}}+2 \mathrm{c}_{0} \mathrm{c}_{1}\left(\frac{h_{i}^{\left(p_{1}+1\right)}}{\mathrm{p}_{1}+1}\right)+2 \mathrm{c}_{0} \mathrm{c}_{2}\left(\frac{h_{i}^{\left(p_{2}+1\right)}}{\mathrm{p}_{2}+1}\right)+\ldots+\right. \\
& 2 \mathrm{c}_{0} \mathrm{c}_{(\mathrm{n}-1)}\left(\frac{h_{i}^{\left(p_{(n-1)}+1\right)}}{\mathrm{p}_{(\mathrm{n}-1)}+1}\right)+2 \mathrm{c}_{0} \mathrm{c}_{\mathrm{n}}\left(\frac{h_{i}{ }^{\left(p_{n}+1\right)}}{\mathrm{p}_{\mathrm{n}}+1}\right)+2 \mathrm{c}_{1} \mathrm{c}_{2}\left(\frac{h_{i}^{\left(p_{1}+\mathrm{p}_{2}+1\right)}}{\mathrm{p}_{1}+\mathrm{p}_{2}+1}\right)+ \\
& \ldots+\mathbf{c}_{1} \mathfrak{c}_{(n-1)}\left(\frac{h_{i}^{\left(p_{1}+p_{(n-1)}+1\right)}}{p_{1}+p_{(n-1)}+1}\right)+2 c_{1} c_{n}\left(\frac{h_{i}^{\left(p_{1}+p_{n}+1\right)}}{p_{1}+p_{n}+1}\right)+2 c_{2} c_{(n-1)}\left(\frac{h_{i}^{\left(p_{2}+p_{(n-1)}+1\right)}}{p_{2}+p_{(n-1)}+1}\right) \\
& +2 \mathrm{c}_{2} \mathrm{c}_{\mathrm{n}}\left(\frac{h_{i}^{\left(p_{2}+p_{n}+1\right)}}{\mathrm{p}_{2}+\mathrm{p}_{\mathrm{n}}+1}\right)+\ldots+2 \mathrm{c}_{(\mathrm{n}-1)} c_{n}\left(\frac{h_{i}^{\left(p_{(n-1)}+\mathrm{p}_{\mathrm{n}}+1\right)}}{\mathrm{p}_{(\mathrm{n}-1)}+\mathrm{p}_{\mathrm{n}}+1}\right)+\mathrm{c}_{1}^{2}\left(\frac{h_{i}^{\left(2 p_{1}+1\right)}}{2 \mathrm{p}_{1}+1}\right) \\
& \left.+\mathrm{c}_{2}^{2}\left(\frac{h_{i}^{\left(2 p_{2}+1\right)}}{2 \mathrm{p}_{2}+1}\right)+\ldots+\mathrm{c}_{(\mathrm{n}-1)}^{2}\left(\frac{h_{i}^{\left(2 p_{(n-1)}+1\right)}}{2 \mathrm{p}_{(\mathrm{n}-1)}}\right)+\mathrm{c}_{\mathrm{n}}^{2}\left(\frac{h_{i}^{\left(2 p_{n}+1\right)}}{2 \mathrm{p}_{\mathrm{n}}+1}\right)\right]_{h_{1}}^{h_{2}}+\varepsilon_{\mathrm{i}}
\end{aligned}
$$

em que: $c_{i s}, p_{i s}, D A P, K, h_{i}$, e å foram definidos anteriormente.

\section{MODELO DE GOULDING \& MURRAY (1976)}

A proposta de Goulding \& Murray foi a de estabelecer uma compatibilidade entre a função de afilamento e a equação de volume. Para isso, procederam à modificaçōes no modelo proposto por Schöepfer (1966) e apresentaram um modelo expresso por:

$$
d_{i}{ }^{2}=\frac{\hat{V}}{K H}\left[\beta_{1}\left(\frac{L}{H_{t}}\right)+\beta_{2}\left(\frac{L}{H_{t}}\right)^{2}+\ldots+\beta_{n}\left(\frac{L}{H_{t}}\right)^{n}\right]+\varepsilon_{i}
$$

em que: $b_{\text {is }}=$ parâmetros a serem estimados; $\mathrm{d}_{\mathrm{i}}=$ diâmetro comercial (cm); $\mathrm{K}=\pi / 40000 ; \hat{\mathrm{V}}=$ volume estimado pela equação de volume para árvore individual $\left(\mathrm{m}^{3}\right) ; \mathrm{H}_{\mathrm{t}}=$ altura total $(\mathrm{m}) ; \mathrm{L}$ $=\left(\mathrm{H}_{\mathrm{t}}-\mathrm{h}_{\mathrm{i}}\right) ; \mathrm{h}_{\mathrm{i}}=$ altura comercial $(\mathrm{m}) ; \mathrm{e}, \varepsilon_{\mathrm{i}}=$ erro de estimativa.

A proposta de Goulding \& Murray (1976) para a função de afilamento exige um prévio ajuste na equação para volume e as estimativas obtidas para cada árvore, visto que a função de afilamento apresentará compatibilidade com a equação de volume. Para este ajuste, foi selecionado o modelo Scolforo (1997), expresso pelo modelo:

$$
\hat{V}=\beta_{0}+\beta_{1}\left(\frac{D A P}{H_{t}}\right)+\beta_{2}\left(D A P^{2} H_{t}\right)+\beta_{3}\left(D A P^{3} H_{t}\right)+\varepsilon_{i}
$$

em que: DAP, $\hat{\mathrm{V}}, \beta_{\mathrm{i},}, \mathrm{H}_{\mathrm{t}}$, e a $\mathrm{a}$ foram definidos anteriormente.

Assim, o volume real advindo da cubagem rigorosa foi empregado para o ajuste do modelo e o volume estimado pelo modelo de Scolforo (1997) foi utilizado para a obtenção das estatísticas de acurácia.

A forma linearizada do polinômio apresentado (7), que possibilita a seleção das variáveis com distintas potências pelo procedimento estatístico Stepwise, é:

$$
\frac{d^{2} K H}{\hat{V}}-\frac{2 \mathrm{~L}}{\mathrm{H}_{\mathrm{t}}}=\beta_{2}^{\prime}\left(3\left(\frac{L}{H_{t}}\right)^{2}-\frac{2 L}{H_{t}}\right)+\beta_{3}^{\prime}\left(4\left(\frac{L}{H_{t}}\right)^{3}-\frac{2 L}{H_{t}}\right)+\beta_{\mathrm{n}}^{\prime}\left((n+1)\left(\frac{L}{H_{t}}\right)^{n}-\frac{2 L}{H_{t}}\right)+\varepsilon_{\mathrm{i}}
$$

Posteriormente ao ajuste deste modelo (linearizado), os coeficientes do modelo original (7) podem ser calculados como:

$$
\beta_{1}=2 \cdot\left(1-\sum_{i=2}^{n} \beta_{i}^{\prime}\right) ; \quad \beta_{2}=3 \cdot \beta_{2}^{\prime} ; \quad \beta_{n}=(n+1) \cdot \beta_{n}^{\prime} .
$$

Resolvendo-se a integral da expressão original (7), obtém-se a fórmula para cálculo dos volumes comerciais. Vale ressaltar que na expressão empregada, a distância do topo da árvore até um ponto h qualquer, os volumes estimados correspondem aos volumes da ponta da árvore até a uma altura h, e não ao volume da base da árvore até uma altura comercial (Scolforo, 1997).

Assim, o volume total ou o dos sortimentos pode ser obtido como:

$$
V c=\frac{\hat{V}}{H_{t}}\left[\frac{\beta_{1} L^{2}}{2 H_{t}}+\frac{\beta_{2} L^{3}}{3 H_{t}^{2}}+\frac{\beta_{3} L^{4}}{4 H_{t}^{3}}+\ldots+\frac{\beta_{n} L^{(n+1)}}{(n+1) H_{t}^{n}}\right]_{0}^{h}+\varepsilon_{i}
$$

em que: $\mathrm{Vc}=$ volume comercial da ponta da árvore até a altura $\mathrm{h}$ $\left.(\mathrm{em} \mathrm{m})^{3}\right) ; \hat{\mathrm{V}}, \mathrm{L}, \beta_{\mathrm{is}}, \mathrm{H}_{\mathrm{t}}$ e $\varepsilon_{\mathrm{i}}$ foram definidos anteriormente.

Os expoentes testados foram: $3 ; 4 ; 5 ; 6 ; 7 ; 8 ; 9 ; 10 ; 11 ; 12$; $13 ; 14 ; 15 ; 16 ; 17 ; 18 ; 19 ; 20 ; 25 ; 30 ; 35 ; 40 ; 45 ; 50 ; 55 ; 60$; 65; 70; 75; 80; 85; 90; e, 95.

\section{PRECISÃO E ACURÁCIA DOS MODELOS DE AFILAMENTO.}

A avaliação da precisão dos modelos de afilamento ajustados foi realizada considerando-se o coeficiente de determinação $\left(\mathrm{R}^{2}\right)$ e o erro padrão da estimativa $\left(\mathrm{S}_{\mathrm{yx}}\right)$. Estas estatísticas permitem verificar se os modelos apresentaram ajustes satisfatórios, de maneira geral.

Também foi avaliada a acurácia, ou seja, a diferença entre o valor estimado (diâmetro ou volume) e o valor real. Para isto, foram adotadas as mesmas estatísticas já utilizadas por Parresol et al. (1987), Figueiredo Filho et al. (1996), Fischer (1997), Scolforo et al. (1998), Assis et al. (2001) e Ferreira (2004). Estas estatísticas possibilitam realizar uma análise mais particularizada do desempenho das estimativas ao longo de todo o fuste, uma vez que podem ser calculadas para cada altura, em que foram tomados os diâmetros por ocasião da cubagem rigorosa.

Os resultados da acurácia dos modelos de afilamento testados foram avaliados através de um ranking construído para o conjunto das árvores. As variáveis consideradas nesta análise foram o diâmetro e o volume em cada posição de medição, os volumes parciais referentes às mesmas posições e, ainda, o volume total, conforme encontrado na Tabela 2 .

Considerando-se as estatísticas desvio (D), desvio padrão das diferenças (SD), soma do quadrado do resíduo relativo (SSRR) e porcentagem dos resíduos $(\mathrm{RP})$, atribui-se a nota quatro à equação que apresentou maior acurácia em cada posição relativa de medição 
e, assim, de forma decrescente, até a nota um para a equação menor acurácia. No caso de mesmo valor em qualquer das estatísticas para diferentes equações, estas receberam a mesma nota. O resultado final do ranking foi definido pela soma das notas de todas as estatísticas; o melhor modelo foi aquele que apresentou maior soma de notas.

Para o modelo de Goulding \& Murray (1976) foram consideradas duas situações na atribuição das notas do ranking. Na primeira situação, as estatísticas foram obtidas considerandose os volumes reais advindos da cubagem rigorosa, possibilitando a simulação de uma situação fictícia em que todas as estimativas

Tabela 2 - Estatísticas utilizadas para a avaliação da acurácia nas estimativas dos diâmetros e volumes de Tectona grandis L.f., em quatro povoamentos na microrregião do Baixo Rio Acre, estado do Acre.

\begin{tabular}{lll}
\hline Estatísticas & Diâmetros & Volumes \\
\hline
\end{tabular}
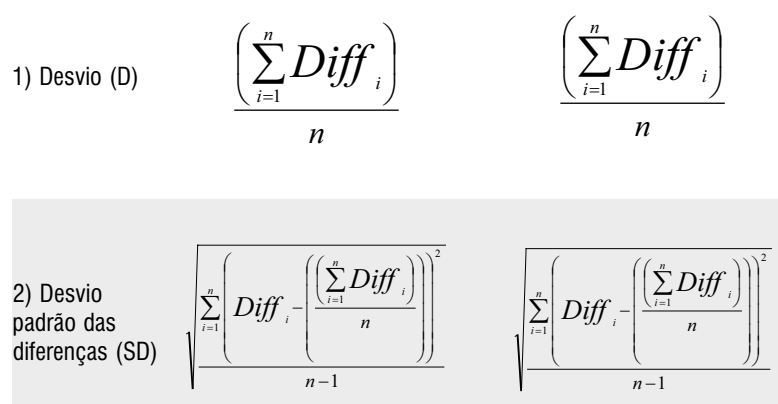

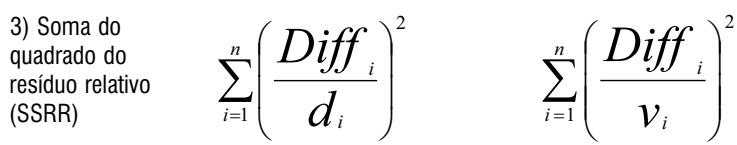

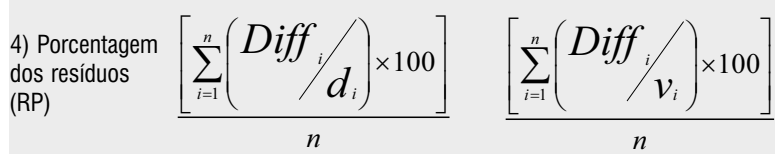

Em que: Diff $=\left(V_{-}-V_{\text {. }}\right)$ ou $\left(\mathrm{d}-\mathrm{d}\right.$ ) é o desvio dos volumes ou dos diâmetros; $\mathrm{V}_{\mathrm{i}}=$ volume total ou comercial até 0 diâmetro $\mathrm{I}$ da árvore; $\mathrm{V}_{\mathrm{ei}}=$ volume total ou comercial estimado; $\mathrm{d}_{\mathrm{i}}$ $=$ diâmetro da árvore na posição i do fuste; $\mathrm{d}_{\mathrm{ei}}{ }_{\mathrm{ei}}=$ diâmetro da árvore estimado para a posição i do fuste e $\mathrm{n}=$ número de árvores. do modelo seriam compatíveis com o volume real das árvores e não com o volume estimado. A segunda situação foi a obtenção das estatísticas considerando-se o volume estimado. Esta é uma situação que simula a condição de campo, na qual se têm os volumes estimados das árvores de uma amostra e não o volume real. Considerando estas duas situaçôes para o modelo de Goulding \& Murray (1976), foram classificados quatro modelos, tendo o modelo com volume real sido apenas considerado para uma situação fictícia e não foi objeto de seleção.

\section{TESTE DE IDENTIDADE DE MODELO.}

Após a seleção da melhor equação de afilamento foi empregado o teste de identidade de modelos. Neste caso, no qual os modelos são lineares, adotou-se o procedimento descrito por Graybill (1976), que consiste na redução da soma dos quadrados. A finalidade da aplicação desta metodologia foi avaliar a necessidade de ajustes por grupos ou individuais, considerando as diferentes áreas estudadas.

Para modelos lineares, o teste de identidade permite observar estatisticamente, por meio do teste $\mathrm{F}$, a significância da diferença entre o total das somas dos quadrados das regressōes ajustadas para cada área de estudo isoladamente (modelo completo - $\Omega$ ) e a soma do quadrado da regressão ajustada para o conjunto das quatro áreas estudadas (modelo reduzido - $w$ ).

A Tabela 3 demonstra o arranjo para proceder ao cálculo das estatísticas da identidade de modelos para regressão linear.

As hipóteses foram definidas da seguinte forma:

- $\mathrm{H}_{0}=$ o modelo reduzido $(w)$ ajustado para o conjunto $\{z\}$ de dados das quatro áreas estudadas é idêntico aos modelos completos ajustados para cada uma das áreas pertencentes a $\{z\}$; e,

- $\mathrm{H}_{1}=$ contrária a $\mathrm{H}_{0}$.

A combinação dos quatro ambientes de produção possibilitou a construção de onze hipóteses para a avaliação do melhor modelo de afilamento.

\section{RESULTADO E DISCUSSÃO}

\section{PRECISÃO DOS MODELOS POLINOMIAIS NA ESTIMATIVA DOS DIÂMETROS.}

Os parâmetros e potências de todos os modelos polinomiais testados e as respectivas estatísticas de precisão encontram-se na

Tabela 3 - Análise de variância para 0 teste de identidade de modelos lineares.

\begin{tabular}{|c|c|c|c|c|}
\hline Fonte de variação & G.L. & S.Q. & Q.M. & $\mathbf{F}$ \\
\hline Modelo completo & $(A \times p)$ & S.Q.Reg. $(\Omega)$ & & \\
\hline Modelo reduzido & $P$ & S.Q.Reg.(w) & & \\
\hline Diferença para testar hipótese & $(A-1) p$ & S.Q.Reg.( $(\Omega)$ - (Reg.(W) & S.Q.(diferença)/ $(A-1) p$ & Q.M.(diferença)/ Q.M.(resíduo) \\
\hline Resíduo & $N-(A \times p)$ & S.Q.Total( $(\Omega)-S . Q . R e g .(\Omega)$ & S.Q.(resíduo)/ N-(Axp) & \\
\hline Total & $\mathrm{N}$. & S.Q.Total $(\Omega)$ & & \\
\hline
\end{tabular}


Tabela 4. Para os modelos de afilamento Hradetzky (1976) e Goulding \& Murray (1976) foi empregado o procedimento Stepwise para a seleção das variáveis e suas respectivas potências.

As estatísticas de precisão dos modelos de afilamento oferecem um indicativo de qualidade dos modelos e demonstram a correlação entre as variáveis independentes e a variável dependente. Porém, não consistem no instrumento mais adequado para a tomada de decisão, sobre qual modelo apresenta o melhor desempenho.

O modelo Schöepfer (1966), apesar de apresentar valores do coeficiente de determinação $\left(\mathrm{R}^{2}\right)$ e erro padrão residual percentual $\left(\mathrm{S}_{\mathrm{yx} \%}\right)$ aceitáveis, foi o modelo que apresentou os piores resultados com $R^{2}$ de $93,37 \%$ e $S_{y x \%}$ de $22,44 \%$.

O modelo Goulding \& Murray (1976) foi o ajuste com melhores resultados de $\mathrm{R}^{2} \mathrm{eS}_{\mathrm{yx} \%}$, seguido pelo modelo Hradetzky (1976).

\section{ACURÁCIA DOS MODELOS PARA ESTIMAR DIÂMETROS AO LONGO DO FUSTE.}

As Tabelas 5, 6, 7 e 8 apresentam as estatísticas de acurácia referente ao resíduo percentual, desvio, soma de quadrados do

Tabela 4 - Parâmetros estimados e medidas de precisão para os modelos polinomiais de Schöepfer (1966), Hradetzky (1976) e Goulding \& Murray (1976), para o conjunto de dados dos quatro povoamentos Tectona grandis L.f. estudados.

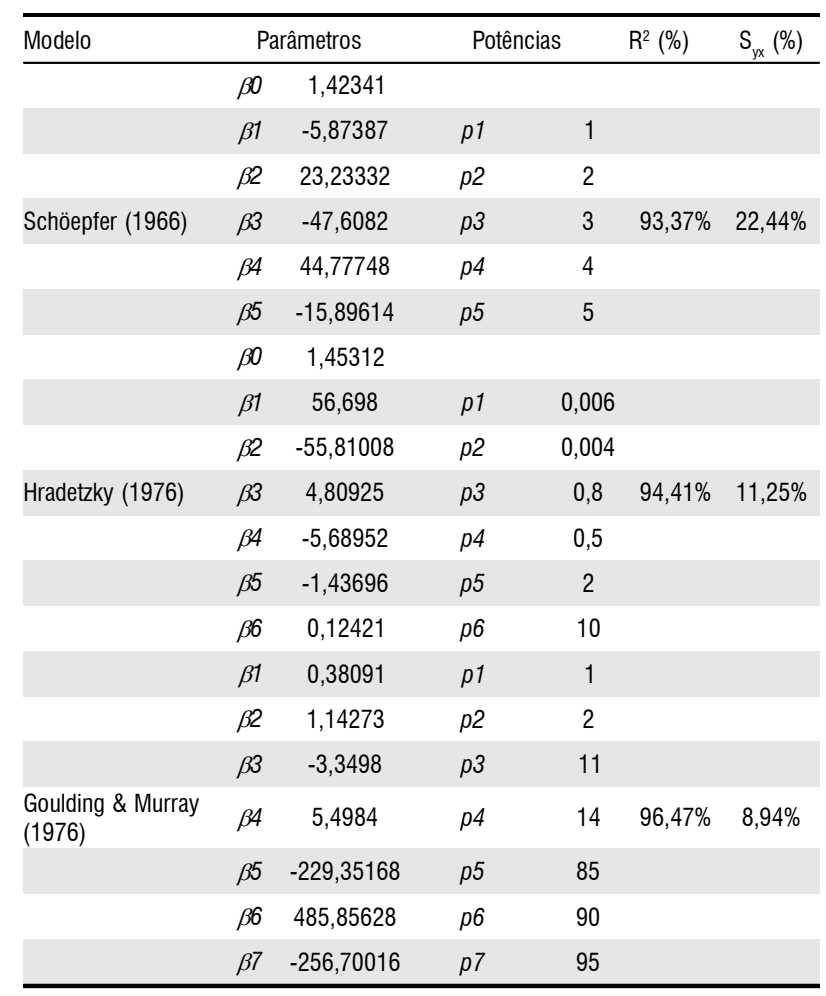

Em que: $\beta_{\mathrm{is}}=$ parâmetros estimados, $\mathrm{p}_{\mathrm{is}}=$ potências selecionadas, $\mathrm{R}^{2}=$ coeficiente de determinação e e $S_{y x} \%=$ erro padrão residual percentual. resíduo relativo, desvio padrão das diferenças e as respectivas notas do ranking, considerando as quatro estatísticas obtidas para os três modelos testados e o modelo Goulding \& Murray (1976) com volume real.

Para a base de dados avaliada, o modelo Schöepfer (1966) apresentou estatísticas que sempre apontaram uma capacidade inferior do modelo de predizer os valores do diâmetro ao longo do fuste. Considerando-se as quatro estatísticas e as 36 posiçōes no fuste, os modelos tiveram 144 oportunidades para pontuar e formar a nota final do ranking. No entanto, o modelo Schöepfer (1966) obteve os piores resultados estatísticos entre os modelos testados, em 66 ocasióes.

O modelo Hradetzky (1976) apresentou resultados estatísticos intermediários. Apesar de apresentar um grande número de estatísticas mal pontuadas, os valores das estatísticas que receberam notas $2 \mathrm{e} 3$ permitiram que o modelo fosse superior ao modelo Schöepfer (1966).

As duas situaçôes testadas para o modelo Goulding \& Murray (1976) apresentaram excelentes estatísticas de acurácia.

A situação em que foi considerado o volume estimado, o modelo Goulding \& Murray (1976) apresentou as melhores estatísticas de acurácia para o diâmetro estimado ao longo do fuste. Em apenas 6,61\% das oportunidades de pontuação, o modelo Goulding \& Murray (1976) recebeu a pior nota; nas demais oportunidades foi o melhor resultado estatístico em $88,97 \%$ das ocasiōes.

No caso do modelo Goulding \& Murray (1976) com volume real (considerado apenas como referência no ranking), destacamse os resultados de desvio (D), cujos valores, para todos os diâmetros ao longo do fuste, foram muito próximos de zero, mostrando que, ao estabelecer uma compatibilidade com o volume real (dados de cubagem), cria-se uma situação fictícia de falsa acurácia, visto que esta situação em condiçōes de trabalho de campo é tecnicamente inviável, ou seja, realizar a cubagem rigorosa de todas as árvores amostradas.

O ranking demonstra que o modelo Hradetzky (1976) obteve melhores estatísticas em duas posiçōes na árvore, na posição de altura absoluta de 6 e 14,5 metros, a primeira posição na porção mediana do fuste e a outra na porção superior no fuste. Nas demais posiçōes do fuste (34 posiçōes), o modelo Goulding \& Murray (1976), com emprego do volume estimado, obteve sempre as melhores notas de ranking.

O fato do modelo Schöepfer (1966) não ter sido selecionado em nenhuma oportunidade é um bom indicativo de que a estratégia de mesclar potências de alto valor e potências menores consiste numa boa alternativa para representar bem o perfil da árvore. Isso porque ao observar os resultados das estatísticas de acurácia e do ranking, revela-se a dificuldade do modelo de Schöepfer (1966) para representar os diâmetros correspondentes 
Tabela 5 - Ranking da acurácia para o modelo Schöepfer (1966) dos diâmetros estimados ao longo do fuste das árvores de Tectona grandis L.f., para 0 conjunto de dados dos quatro povoamentos estudados.

\begin{tabular}{|c|c|c|c|c|c|c|c|c|c|}
\hline \multirow[b]{2}{*}{ hi } & \multicolumn{4}{|c|}{ Estatísticas de acurácia } & \multicolumn{5}{|c|}{ Ranking } \\
\hline & $\mathrm{RP}$ & $\mathrm{D}$ & SSRR & $\mathrm{DP}$ & $\mathrm{RP}$ & $D$ & SSRR & $\mathrm{DP}$ & Nota ranking. $h_{i}^{-1}$ \\
\hline 0,00 & 0,7665 & 0,0327 & 1,9574 & 1,7986 & 4,0 & 3,0 & 2,0 & 2,0 & 11,0 \\
\hline 0,25 & 0,6673 & $-0,0281$ & 0,5759 & 1,2189 & 4,0 & 4,0 & 2,0 & 1,0 & 11,0 \\
\hline 0,50 & $-1,5350$ & $-0,3474$ & 1,1526 & 1,1340 & 1,0 & 1,0 & 1,0 & 1,0 & 4,0 \\
\hline 0,75 & $-1,3080$ & $-0,3071$ & 0,5332 & 0,9787 & 1,0 & 1,0 & 1,0 & 2,0 & 5,0 \\
\hline 1,00 & $-0,7231$ & $-0,2234$ & 0,7439 & 0,7997 & 2,0 & 1,0 & 1,0 & 2,0 & 6,0 \\
\hline 1,30 & 0,6654 & $-0,0929$ & 0,5880 & 0,6386 & 1,0 & 2,0 & 1,0 & 1,0 & 5,0 \\
\hline 1,50 & $-0,4521$ & $-0,2034$ & 0,4677 & 0,8116 & 3,0 & 2,0 & 1,0 & 1,0 & 7,0 \\
\hline 2,00 & 1,9956 & 0,0499 & 1,0959 & 0,8462 & 1,0 & 4,0 & 1,0 & 2,0 & 8,0 \\
\hline 2,50 & 0,2330 & $-0,0808$ & 0,8014 & 1,0203 & 4,0 & 2,0 & 2,0 & 2,0 & 10,0 \\
\hline 3,00 & 2,3143 & 0,1065 & 1,2617 & 0,8258 & 1,0 & 2,0 & 2,0 & 2,0 & 7,0 \\
\hline 3,50 & $-0,8746$ & $-0,1523$ & 0,9550 & 0,9986 & 4,0 & 2,0 & 2,0 & 2,0 & 10,0 \\
\hline 4,00 & 0,8760 & $-0,0370$ & 1,7178 & 0,8809 & 2,0 & 2,0 & 2,0 & 2,0 & 8,0 \\
\hline 4,50 & $-1,7850$ & $-0,2595$ & 1,2074 & 0,9799 & 1,0 & 1,0 & 1,0 & 2,0 & 5,0 \\
\hline 5,00 & 1,7509 & $-0,0258$ & 2,5875 & 0,8906 & 2,0 & 4,0 & 1,0 & 1,0 & 8,0 \\
\hline 5,50 & $-0,0903$ & $-0,1898$ & 1,8699 & 1,0579 & 4,0 & 1,0 & 1,0 & 1,0 & 7,0 \\
\hline 6,00 & $-0,0148$ & $-0,1239$ & 2,5189 & 0,9442 & 4,0 & 1,0 & 1,0 & 1,0 & 7,0 \\
\hline 6,50 & $-3,0350$ & $-0,2986$ & 1,5571 & 0,9734 & 2,0 & 1,0 & 1,0 & 1,0 & 5,0 \\
\hline 7,00 & $-1,3330$ & $-0,1634$ & 2,8875 & 1,0048 & 2,0 & 1,0 & 1,0 & 1,0 & 5,0 \\
\hline 7,50 & $-2,3150$ & $-0,2528$ & 1,8131 & 1,0687 & 2,0 & 2,0 & 1,0 & 1,0 & 6,0 \\
\hline 8,00 & $-0,3096$ & $-0,1267$ & 2,7723 & 0,9207 & 4,0 & 2,0 & 2,0 & 2,0 & 10,0 \\
\hline 8,50 & $-3,4440$ & $-0,2759$ & 1,2539 & 0,8335 & 2,0 & 2,0 & 2,0 & 2,0 & 8,0 \\
\hline 9,00 & $-2,8540$ & $-0,2147$ & 2,0908 & 0,8162 & 2,0 & 2,0 & 1,0 & 2,0 & 7,0 \\
\hline 9,50 & $-5,1290$ & $-0,2829$ & 1,2179 & 0,7940 & 2,0 & 2,0 & 2,0 & 2,0 & 8,0 \\
\hline 10,00 & $-7,9170$ & $-0,4182$ & 3,6942 & 0,9014 & 2,0 & 2,0 & 1,0 & 1,0 & 6,0 \\
\hline 10,50 & $-8,6600$ & $-0,4437$ & 1,3180 & 0,7762 & 1,0 & 2,0 & 1,0 & 2,0 & 6,0 \\
\hline 11,00 & $-8,5630$ & $-0,3778$ & 2,4685 & 0,7588 & 2,0 & 1,0 & 2,0 & 2,0 & 7,0 \\
\hline 11,50 & $-9,7430$ & $-0,4612$ & 1,0409 & 0,7686 & 1,0 & 1,0 & 1,0 & 2,0 & 5,0 \\
\hline 12,00 & $-13,0000$ & $-0,4335$ & 3,7907 & 0,8012 & 1,0 & 1,0 & 1,0 & 1,0 & 4,0 \\
\hline 12,50 & $-16,5700$ & $-0,6858$ & 1,5277 & 0,7825 & 1,0 & 1,0 & 1,0 & 1,0 & 4,0 \\
\hline 13,00 & $-23,8900$ & $-0,7343$ & 2,7423 & 0,6996 & 1,0 & 1,0 & 1,0 & 1,0 & 4,0 \\
\hline 13,50 & $-28,8400$ & $-1,0270$ & 0,8754 & 0,7537 & 1,0 & 1,0 & 1,0 & 1,0 & 4,0 \\
\hline 14,00 & $-38,7800$ & $-0,9062$ & 2,4277 & 0,7340 & 2,0 & 2,0 & 2,0 & 1,0 & 7,0 \\
\hline 14,50 & $-4,8780$ & $-0,2512$ & 0,0024 & . & 2,0 & 2,0 & 2,0 & 0,0 & 6,0 \\
\hline 15,00 & $-31,1200$ & $-0,5852$ & 0,2790 & 0,1831 & 2,0 & 2,0 & 2,0 & 4,0 & 10,0 \\
\hline 15,50 & $-13,0000$ & $-0,4745$ & 0,0169 & . & 1,0 & 1,0 & 1,0 & 0,0 & 3,0 \\
\hline 16,00 & $-7,0140$ & $-0,2174$ & 0,0049 & . & 2,0 & 2,0 & 2,0 & 0,0 & 6,0 \\
\hline Total & & & & & & & & & 240,0 \\
\hline
\end{tabular}

Em que: $h_{i}=$ altura ao longo do fuste, $R P=$ resíduo percentual, $D=$ desvio, SSRR = soma de quadrado do resíduo relativo e $D P=$ desvio padrão das diferenças. 
Tabela 6 - Ranking da acurácia para o modelo Hradetzky (1976) dos diâmetros estimados ao longo do fuste das árvores de Tectona grandis L.f., para 0 conjunto de dados dos quatro povoamentos estudados.

\begin{tabular}{|c|c|c|c|c|c|c|c|c|c|}
\hline \multirow[b]{2}{*}{ hi } & \multicolumn{4}{|c|}{ Estatísticas de acurácia } & \multicolumn{5}{|c|}{ Ranking } \\
\hline & $\mathrm{RP}$ & $\mathrm{D}$ & SSRR & $\mathrm{DP}$ & $\mathrm{RP}$ & $\mathrm{D}$ & SSRR & $\mathrm{DP}$ & Nota ranking. $h_{i}^{-1}$ \\
\hline 0,00 & $-1,3050$ & $-0,2912$ & 2,0574 & 1,8506 & 2,0 & 1,0 & 1,0 & 1,0 & 5,0 \\
\hline 0,25 & 2,0969 & 0,1691 & 0,6267 & 1,2120 & 1,0 & 1,0 & 1,0 & 2,0 & 5,0 \\
\hline 0,50 & 0,5096 & $-0,0777$ & 1,0520 & 1,0723 & 4,0 & 3,0 & 2,0 & 2,0 & 11,0 \\
\hline 0,75 & 0,2201 & $-0,1010$ & 0,4503 & 0,9027 & 2,0 & 2,0 & 2,0 & 4,0 & 10,0 \\
\hline 1,00 & $-0,1030$ & $-0,1263$ & 0,5904 & 0,7191 & 4,0 & 2,0 & 2,0 & 4,0 & 12,0 \\
\hline 1,30 & 0,3148 & $-0,1067$ & 0,4420 & 0,5598 & 3,0 & 1,0 & 2,0 & 2,0 & 8,0 \\
\hline 1,50 & $-1,2810$ & $-0,2726$ & 0,4211 & 0,7698 & 1,0 & 1,0 & 2,0 & 2,0 & 6,0 \\
\hline 2,00 & 0,5886 & $-0,0898$ & 1,0622 & 0,8688 & 3,0 & 2,0 & 2,0 & 1,0 & 8,0 \\
\hline 2,50 & $-1,0620$ & $-0,2188$ & 0,8892 & 1,0704 & 1,0 & 1,0 & 1,0 & 1,0 & 4,0 \\
\hline 3,00 & 1,6694 & 0,0219 & 1,4292 & 0,8870 & 2,0 & 4,0 & 1,0 & 1,0 & 8,0 \\
\hline 3,50 & $-0,9366$ & $-0,1814$ & 1,0557 & 1,0518 & 3,0 & 1,0 & 1,0 & 1,0 & 6,0 \\
\hline 4,00 & 1,2989 & $-0,0162$ & 1,8166 & 0,9091 & 1,0 & 4,0 & 1,0 & 1,0 & 7,0 \\
\hline 4,50 & $-1,0790$ & $-0,2050$ & 1,1830 & 0,9861 & 3,0 & 2,0 & 2,0 & 1,0 & 8,0 \\
\hline 5,00 & 2,3980 & 0,0360 & 2,4398 & 0,8639 & 1,0 & 3,0 & 2,0 & 2,0 & 8,0 \\
\hline 5,50 & 0,5217 & $-0,1283$ & 1,7012 & 1,0109 & 3,0 & 2,0 & 2,0 & 2,0 & 9,0 \\
\hline 6,00 & 0,3273 & $-0,0753$ & 2,2410 & 0,8896 & 3,0 & 3,0 & 2,0 & 2,0 & 10,0 \\
\hline 6,50 & $-3,0600$ & $-0,2743$ & 1,4596 & 0,9212 & 1,0 & 2,0 & 2,0 & 2,0 & 7,0 \\
\hline 7,00 & $-1,5700$ & $-0,1570$ & 2,7503 & 0,9644 & 1,0 & 2,0 & 2,0 & 2,0 & 7,0 \\
\hline 7,50 & $-2,6530$ & $-0,2647$ & 1,8014 & 1,0412 & 1,0 & 1,0 & 2,0 & 2,0 & 6,0 \\
\hline 8,00 & $-0,6381$ & $-0,1510$ & 2,8736 & 0,9220 & 3,0 & 1,0 & 1,0 & 1,0 & 6,0 \\
\hline 8,50 & $-4,1580$ & $-0,3224$ & 1,3143 & 0,8417 & 1,0 & 1,0 & 1,0 & 1,0 & 4,0 \\
\hline 9,00 & $-3,8080$ & $-0,2746$ & 2,0655 & 0,8307 & 1,0 & 1,0 & 2,0 & 1,0 & 5,0 \\
\hline 9,50 & $-5,5060$ & $-0,3272$ & 1,2708 & 0,8268 & 1,0 & 1,0 & 1,0 & 1,0 & 4,0 \\
\hline 10,00 & $-9,7620$ & $-0,4844$ & 3,3094 & 0,9008 & 1,0 & 1,0 & 2,0 & 2,0 & 6,0 \\
\hline 10,50 & $-8,2120$ & $-0,4523$ & 1,2832 & 0,8151 & 2,0 & 1,0 & 2,0 & 1,0 & 6,0 \\
\hline 11,00 & $-8,5830$ & $-0,3737$ & 2,6517 & 0,7788 & 1,0 & 2,0 & 1,0 & 1,0 & 5,0 \\
\hline 11,50 & $-8,0430$ & $-0,4027$ & 0,9691 & 0,7925 & 2,0 & 2,0 & 2,0 & 1,0 & 7,0 \\
\hline 12,00 & $-10,8800$ & $-0,3455$ & 3,1648 & 0,7923 & 2,0 & 2,0 & 3,0 & 2,0 & 9,0 \\
\hline 12,50 & $-13,8600$ & $-0,5820$ & 1,0103 & 0,6692 & 2,0 & 2,0 & 2,0 & 2,0 & 8,0 \\
\hline 13,00 & $-21,0600$ & $-0,6082$ & 2,6195 & 0,6860 & 2,0 & 2,0 & 2,0 & 2,0 & 8,0 \\
\hline 13,50 & $-23,8000$ & $-0,8458$ & 0,6717 & 0,7305 & 2,0 & 2,0 & 2,0 & 2,0 & 8,0 \\
\hline 14,00 & $-45,5200$ & $-0,9739$ & 2,5412 & 0,5150 & 1,0 & 1,0 & 1,0 & 4,0 & 7,0 \\
\hline 14,50 & 0,2196 & 0,0113 & 0,0000 & . & 4,0 & 4,0 & 4,0 & 0,0 & 12,0 \\
\hline 15,00 & $-44,3900$ & $-0,6814$ & 0,7107 & 0,6799 & 1,0 & 1,0 & 1,0 & 1,0 & 4,0 \\
\hline 15,50 & $-9,3520$ & $-0,3414$ & 0,0088 & . & 2,0 & 2,0 & 2,0 & 0,0 & 6,0 \\
\hline 16,00 & $-12,2300$ & $-0,3792$ & 0,0150 & . & 1,0 & 1,0 & 1,0 & 0,0 & 3,0 \\
\hline Total & & & & & & & & & 253,0 \\
\hline
\end{tabular}

Em que: $h_{i}, R P, D, S S R R$ e $D P$ foram definidos anteriormente. 
Tabela 7 - Ranking da acurácia para o modelo Goulding \& Murray (1976) aplicado com volume estimado, para os diâmetros estimados ao longo do fuste das árvores de Tectona grandis L.f., para o conjunto de dados dos quatro povoamentos estudados.

\begin{tabular}{|c|c|c|c|c|c|c|c|c|c|}
\hline \multirow[b]{2}{*}{ hi } & \multicolumn{4}{|c|}{ Estatísticas de acurácia } & \multicolumn{5}{|c|}{ Ranking } \\
\hline & $\mathrm{RP}$ & $\mathrm{D}$ & SSRR & $\mathrm{DP}$ & $\mathrm{RP}$ & $\mathrm{D}$ & SSRR & $\mathrm{DP}$ & Nota ranking. $h_{i}^{-1}$ \\
\hline 0,00 & $-0,9058$ & $-0,0125$ & 1,6041 & 1,5514 & 3,0 & 4,0 & 3,0 & 3,0 & 13,0 \\
\hline 0,25 & $-0,7932$ & $-0,0649$ & 0,4560 & 1,0620 & 2,0 & 2,0 & 3,0 & 4,0 & 11,0 \\
\hline 0,50 & $-0,8980$ & $-0,0625$ & 0,7196 & 0,9416 & 3,0 & 4,0 & 4,0 & 4,0 & 15,0 \\
\hline 0,75 & $-0,2041$ & 0,0414 & 0,3717 & 0,9430 & 3,0 & 4,0 & 3,0 & 3,0 & 13,0 \\
\hline 1,00 & $-0,6537$ & $-0,0107$ & 0,4058 & 0,7421 & 3,0 & 4,0 & 4,0 & 3,0 & 14,0 \\
\hline 1,30 & 0,2973 & 0,0631 & 0,1584 & 0,4088 & 4,0 & 3,0 & 4,0 & 4,0 & 15,0 \\
\hline 1,50 & $-0,4585$ & $-0,0135$ & 0,2227 & 0,5476 & 2,0 & 3,0 & 3,0 & 3,0 & 11,0 \\
\hline 2,00 & 0,6920 & 0,0915 & 0,4435 & 0,5970 & 2,0 & 1,0 & 3,0 & 3,0 & 9,0 \\
\hline 2,50 & $-0,7414$ & $-0,0215$ & 0,4719 & 0,8019 & 2,0 & 3,0 & 3,0 & 3,0 & 11,0 \\
\hline 3,00 & 0,9309 & 0,1129 & 0,7020 & 0,6845 & 3,0 & 1,0 & 3,0 & 3,0 & 10,0 \\
\hline 3,50 & $-1,2600$ & $-0,0624$ & 0,6370 & 0,7809 & 1,0 & 3,0 & 3,0 & 3,0 & 10,0 \\
\hline 4,00 & 0,3843 & 0,0435 & 1,0181 & 0,6871 & 3,0 & 1,0 & 3,0 & 3,0 & 10,0 \\
\hline 4,50 & $-1,2990$ & $-0,0904$ & 0,7251 & 0,7056 & 2,0 & 3,0 & 3,0 & 3,0 & 11,0 \\
\hline 5,00 & 1,7457 & 0,1161 & 1,4810 & 0,6391 & 3,0 & 1,0 & 3,0 & 3,0 & 10,0 \\
\hline 5,50 & 0,8972 & 0,0201 & 1,0182 & 0,7620 & 2,0 & 4,0 & 3,0 & 3,0 & 12,0 \\
\hline 6,00 & 1,0372 & 0,0801 & 1,4361 & 0,6907 & 1,0 & 2,0 & 3,0 & 3,0 & 9,0 \\
\hline 6,50 & $-1,9390$ & $-0,0798$ & 0,9862 & 0,6637 & 3,0 & 3,0 & 3,0 & 3,0 & 12,0 \\
\hline 7,00 & $-0,3300$ & 0,0345 & 2,0560 & 0,7576 & 4,0 & 3,0 & 3,0 & 3,0 & 13,0 \\
\hline 7,50 & $-0,8616$ & $-0,0321$ & 1,2107 & 0,7282 & 3,0 & 3,0 & 3,0 & 3,0 & 12,0 \\
\hline 8,00 & 0,8216 & 0,0566 & 2,0438 & 0,6822 & 2,0 & 3,0 & 3,0 & 3,0 & 11,0 \\
\hline 8,50 & $-1,9580$ & $-0,0697$ & 0,9231 & 0,6258 & 3,0 & 3,0 & 3,0 & 3,0 & 12,0 \\
\hline 9,00 & $-1,1500$ & $-0,0348$ & 1,5988 & 0,6691 & 3,0 & 3,0 & 3,0 & 3,0 & 12,0 \\
\hline 9,50 & $-2,7730$ & $-0,0706$ & 0,9831 & 0,6356 & 3,0 & 3,0 & 3,0 & 3,0 & 12,0 \\
\hline 10,00 & $-5,6060$ & $-0,2591$ & 2,9248 & 0,7646 & 3,0 & 3,0 & 3,0 & 3,0 & 12,0 \\
\hline 10,50 & $-5,4160$ & $-0,2396$ & 0,8349 & 0,5977 & 3,0 & 3,0 & 3,0 & 3,0 & 12,0 \\
\hline 11,00 & $-6,0200$ & $-0,2267$ & 1,9375 & 0,6681 & 4,0 & 4,0 & 3,0 & 3,0 & 14,0 \\
\hline 11,50 & $-5,5040$ & $-0,2256$ & 0,6352 & 0,6111 & 3,0 & 4,0 & 3,0 & 3,0 & 13,0 \\
\hline 12,00 & $-8,8750$ & $-0,2399$ & 3,0470 & 0,7321 & 4,0 & 4,0 & 4,0 & 3,0 & 15,0 \\
\hline 12,50 & $-10,1900$ & $-0,4157$ & 0,8856 & 0,6417 & 3,0 & 3,0 & 3,0 & 3,0 & 12,0 \\
\hline 13,00 & $-17,4300$ & $-0,4837$ & 1,9621 & 0,6437 & 3,0 & 3,0 & 3,0 & 3,0 & 12,0 \\
\hline 13,50 & $-21,2400$ & $-0,7153$ & 0,5707 & 0,7271 & 4,0 & 4,0 & 4,0 & 3,0 & 15,0 \\
\hline 14,00 & $-31,1200$ & $-0,6775$ & 1,8726 & 0,7029 & 3,0 & 3,0 & 3,0 & 2,0 & 11,0 \\
\hline 14,50 & 5,6956 & 0,2933 & 0,0032 & . & 1,0 & 1,0 & 1,0 & 0,0 & 3,0 \\
\hline 15,00 & $-22,4900$ & $-0,2911$ & 0,2145 & 0,4922 & 3,0 & 4,0 & 3,0 & 2,0 & 12,0 \\
\hline 15,50 & $-0,7602$ & $-0,0277$ & 0,0001 & . & 4,0 & 4,0 & 4,0 & 0,0 & 12,0 \\
\hline 16,00 & 3,9133 & 0,1213 & 0,0015 & . & 3,0 & 3,0 & 3,0 & 0,0 & 9,0 \\
\hline Total & & & & & & & & & 420,0 \\
\hline
\end{tabular}

Em que: $h_{i}, R P, D, S S R R$ e $D P$ foram definidos anteriormente. 
Tabela 8 - Ranking da acurácia para o modelo Goulding \& Murray (1976) aplicado com volume real, para os diâmetros estimados ao longo do fuste das árvores de Tectona grandis L.f., para 0 conjunto de dados dos quatro povoamentos estudados.

\begin{tabular}{|c|c|c|c|c|c|c|c|c|c|}
\hline \multirow[b]{2}{*}{ hi } & \multicolumn{4}{|c|}{ Estatísticas de acurácia } & \multicolumn{5}{|c|}{ Ranking } \\
\hline & $\mathrm{RP}$ & $\mathrm{D}$ & SSRR & $\mathrm{DP}$ & $\mathrm{RP}$ & $\mathrm{D}$ & SSRR & $\mathrm{DP}$ & Nota ranking. $\mathrm{h}_{\mathrm{i}}^{-1}$ \\
\hline 0,00 & $-1,4270$ & $-0,0654$ & 1,5099 & 1,5066 & 1,0 & 2,0 & 4,0 & 4,0 & 11,0 \\
\hline 0,25 & $-0,7392$ & $-0,0369$ & 0,4127 & 1,1275 & 3,0 & 3,0 & 4,0 & 3,0 & 13,0 \\
\hline 0,50 & $-1,4720$ & $-0,1036$ & 0,7832 & 1,0419 & 2,0 & 2,0 & 3,0 & 3,0 & 10,0 \\
\hline 0,75 & $-0,1776$ & 0,0653 & 0,3691 & 1,0795 & 4,0 & 3,0 & 4,0 & 1,0 & 12,0 \\
\hline 1,00 & $-1,2550$ & $-0,0460$ & 0,5371 & 0,8790 & 1,0 & 3,0 & 3,0 & 1,0 & 8,0 \\
\hline 1,30 & $-0,3151$ & 0,0299 & 0,3342 & 0,5557 & 2,0 & 4,0 & 3,0 & 3,0 & 12,0 \\
\hline 1,50 & $-0,3765$ & 0,0074 & 0,1284 & 0,4314 & 4,0 & 4,0 & 4,0 & 4,0 & 16,0 \\
\hline 2,00 & 0,1895 & 0,0611 & 0,2715 & 0,4550 & 4,0 & 3,0 & 4,0 & 4,0 & 15,0 \\
\hline 2,50 & $-0,5845$ & $-0,0028$ & 0,2300 & 0,5365 & 3,0 & 4,0 & 4,0 & 4,0 & 15,0 \\
\hline 3,00 & 0,4963 & 0,0858 & 0,3053 & 0,4665 & 4,0 & 3,0 & 4,0 & 4,0 & 15,0 \\
\hline 3,50 & $-1,0640$ & $-0,0446$ & 0,3122 & 0,5043 & 2,0 & 4,0 & 4,0 & 4,0 & 14,0 \\
\hline 4,00 & $-0,0183$ & 0,0201 & 0,5143 & 0,4804 & 4,0 & 3,0 & 4,0 & 4,0 & 15,0 \\
\hline 4,50 & $-1,0520$ & $-0,0731$ & 0,3080 & 0,4484 & 4,0 & 4,0 & 4,0 & 4,0 & 16,0 \\
\hline 5,00 & 1,4206 & 0,0968 & 0,7849 & 0,4324 & 4,0 & 2,0 & 4,0 & 4,0 & 14,0 \\
\hline 5,50 & 1,1414 & 0,0353 & 0,5375 & 0,5220 & 1,0 & 3,0 & 4,0 & 4,0 & 12,0 \\
\hline 6,00 & 0,8747 & 0,0672 & 0,7563 & 0,4772 & 2,0 & 4,0 & 4,0 & 4,0 & 14,0 \\
\hline 6,50 & $-1,4110$ & $-0,0564$ & 0,5763 & 0,4881 & 4,0 & 4,0 & 4,0 & 4,0 & 16,0 \\
\hline 7,00 & $-0,3707$ & 0,0265 & 1,3615 & 0,5995 & 3,0 & 4,0 & 4,0 & 4,0 & 15,0 \\
\hline 7,50 & $-0,2819$ & $-0,0100$ & 0,8138 & 0,5866 & 4,0 & 4,0 & 4,0 & 4,0 & 16,0 \\
\hline 8,00 & 0,8809 & 0,0538 & 1,5171 & 0,5608 & 1,0 & 4,0 & 4,0 & 4,0 & 13,0 \\
\hline 8,50 & $-1,1800$ & $-0,0422$ & 0,6056 & 0,4885 & 4,0 & 4,0 & 4,0 & 4,0 & 16,0 \\
\hline 9,00 & $-0,7945$ & $-0,0286$ & 1,2110 & 0,5580 & 4,0 & 4,0 & 4,0 & 4,0 & 16,0 \\
\hline 9,50 & $-2,0000$ & $-0,0464$ & 0,7270 & 0,5958 & 4,0 & 4,0 & 4,0 & 4,0 & 16,0 \\
\hline 10,00 & $-5,0530$ & $-0,2538$ & 2,1689 & 0,6275 & 4,0 & 4,0 & 4,0 & 4,0 & 16,0 \\
\hline 10,50 & $-4,7420$ & $-0,2253$ & 0,5686 & 0,5056 & 4,0 & 4,0 & 4,0 & 4,0 & 16,0 \\
\hline 11,00 & $-6,2560$ & $-0,2420$ & 1,7664 & 0,6033 & 3,0 & 3,0 & 4,0 & 4,0 & 14,0 \\
\hline 11,50 & $-5,4320$ & $-0,2267$ & 0,6142 & 0,5738 & 4,0 & 3,0 & 4,0 & 4,0 & 15,0 \\
\hline 12,00 & $-9,3540$ & $-0,2562$ & 3,2044 & 0,7247 & 3,0 & 3,0 & 2,0 & 4,0 & 12,0 \\
\hline 12,50 & $-9,7240$ & $-0,3989$ & 0,8092 & 0,6024 & 4,0 & 4,0 & 4,0 & 4,0 & 16,0 \\
\hline 13,00 & $-16,5100$ & $-0,4566$ & 1,8352 & 0,5959 & 4,0 & 4,0 & 4,0 & 4,0 & 16,0 \\
\hline 13,50 & $-22,8600$ & $-0,7797$ & 0,6433 & 0,7262 & 3,0 & 3,0 & 3,0 & 4,0 & 13,0 \\
\hline 14,00 & $-30,2300$ & $-0,6605$ & 1,7273 & 0,6377 & 4,0 & 4,0 & 4,0 & 3,0 & 15,0 \\
\hline 14,50 & 3,0645 & 0,1578 & 0,0009 & . & 3,0 & 3,0 & 3,0 & 0,0 & 9,0 \\
\hline 15,00 & $-21,8800$ & $-0,3235$ & 0,1792 & 0,3685 & 4,0 & 3,0 & 4,0 & 3,0 & 14,0 \\
\hline 15,50 & $-3,5710$ & $-0,1304$ & 0,0013 & . & 3,0 & 3,0 & 3,0 & 0,0 & 9,0 \\
\hline 16,00 & 1,2326 & 0,0382 & 0,0002 & . & 4,0 & 4,0 & 4,0 & 0,0 & 12,0 \\
\hline Total & & & & & & & & & 497,0 \\
\hline
\end{tabular}

Em que: $h_{i}, R P, D, S S R R$ e $D P$ foram definidos anteriormente. 
à base da árvore, em que o referido modelo obteve os piores desempenhos de acurácia.

A inserção de potências altas e menores, selecionadas pelo processo estatístico Stepwise garantiu aos modelos Goulding \& Murray (1976) e Hradetzky (1976) os melhores desempenhos de estimativas de diâmetros ao longo do fuste.

A Figura 1 demonstra o perfil médio das árvores, considerando os diâmetros médios reais e estimados para a base de dados estudada e o conjunto dos quatro povoamentos de Tectona grandis L.f.

Os perfis apresentados para os modelos testados, revelam a excelente acurácia para as estimativas do modelo Goulding \&
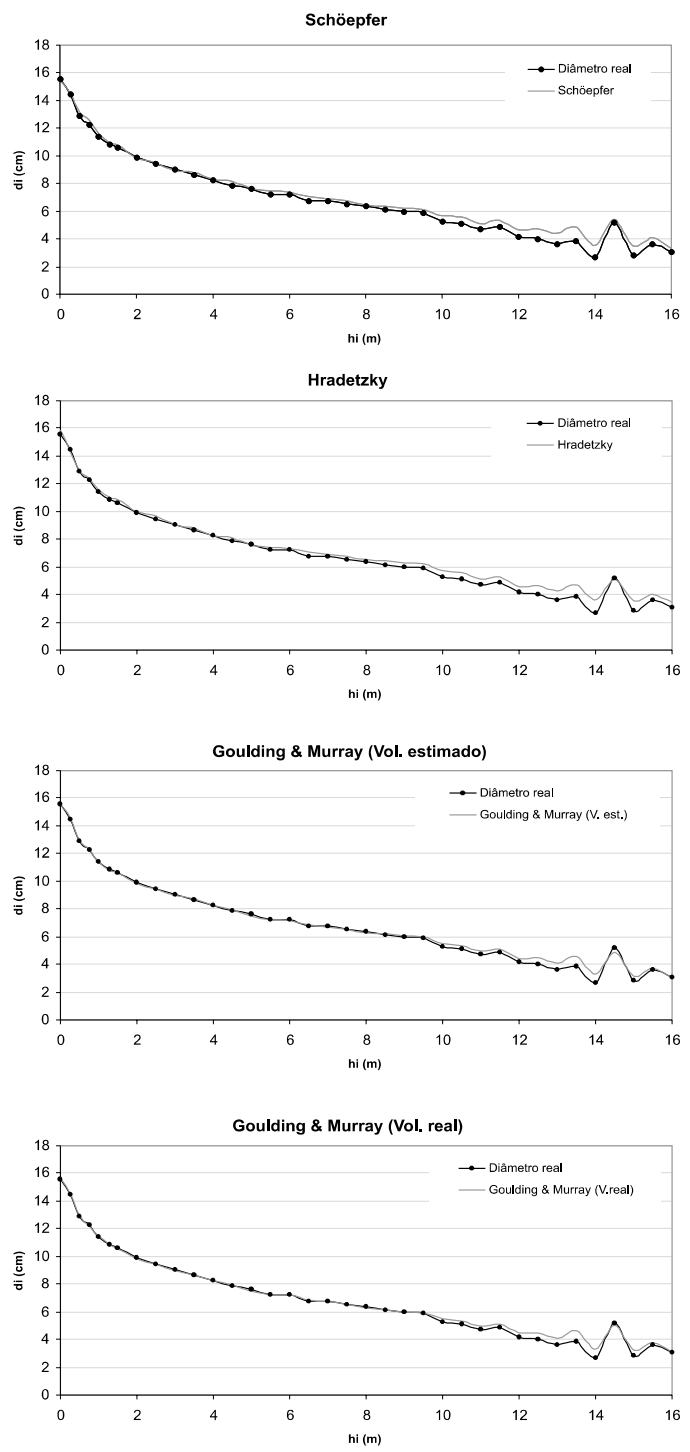

Figura 1 - Diâmetros médios reais e estimados pelos modelos polinomiais de Schöepfer (1966), Hradetzky (1976) e Goulding \& Murray (1976), para 0 conjunto de dados dos quatro povoamentos de Tectona grandis L.f.
Murray (1976) com volume estimado e com o volume real. Para os diâmetros nas maiores alturas, houve dificuldade de estimativa.

O fato de um menor número de árvores alcançarem as maiores alturas provocou a oscilação do perfil nas alturas entre 13,5 e 16 metros em todos os modelos testados.

O perfil médio que representa o resultado do ajuste do modelo Schöepfer (1966) demonstra dificuldade para estimar os valores da base e da ponta do fuste. Percebe-se, que na ponta do fuste ocorre uma instabilidade das estimativas para os diâmetros entre as alturas zero e dois metros, resultado já apontado pelas estatísticas de acurácia.

O perfil médio retratado pelo modelo Hradetzky (1976) apresentou desempenho muito semelhante ao modelo de Goulding \& Murray (1976) com volume estimado, porém o polinômio de potências inteiras e fracionárias distancia mais a linha das estimativas desde a altura de 8,5 metros.

\section{ACURÁCIA DOS MODELOS PARA ESTIMAR VOLUMES AO LONGO} DO FUSTE.

Quando foram calculadas as estimativas de volumes, os três modelos testados mantiveram as mesmas tendências de desempenho alcançadas nas estimativas de diâmetros ao longo do fuste. Contudo, os piores e melhores resultados foram destacados.

As Tabelas 9, 10, 11 e 12 apresentam os resultados das estatísticas de acurácia para os modelos testados e as notas atribuídas para cada estatística no ranking. Os mesmos critérios estabelecidos para as estimativas dos diâmetros foram mantidos para avaliar as estimativas de volume ao longo das diversas posiçôes no fuste. Nos resultados das estatísticas de acurácia para volume de todos os modelos testados na altura 0,00 foi atribuído o valor 0,0 (zero) e a nota de ranking 1,0 , pois não existe volume de fuste na altura 0,00 metro.

O pior desempenho do modelo Schöepfer (1966) foi para as estimativas de volumes ao longo do fuste, pois, em $67,36 \%$ das possibilidades de pontuação no ranking das quatro estatísticas de acurácia avaliadas, o modelo obteve a pior nota. Somente conseguiu a melhor nota em uma estatística numa única posição. De maneira geral, o modelo obteve os piores resultados de volume estimado em qualquer porção do fuste.

O modelo Hradetzky (1976) obteve notas com valor 2,0 e $3,0 \mathrm{em} 77,78 \%$ dos casos avaliados, o que indica uma posição de performance intermediária entre os modelos testados. Contudo, vale ressaltar o excelente desempenho das estimativas de volume para as porçōes na base do fuste, tendo, na posição $0,75 \mathrm{~m}$ de altura a estatística do desvio (D) sido zero e nas alturas 0,25 , 0,50, 1,00, 1,30, 1,50 e 2,00 os valores foram próximo de zero.

O modelo Goulding \& Murray (1976) com volume estimado obteve o melhor desempenho das estatísticas de acurácia para estimativas de volumes ao longo do fuste; $90,97 \%$ das notas 
Tabela 9 - Ranking da acurácia para o modelo Schöepfer (1966) para os volumes estimados ao longo do fuste das árvores de Tectona grandis L.f., para 0 conjunto de dados dos quatro povoamentos estudados.

\begin{tabular}{|c|c|c|c|c|c|c|c|c|c|}
\hline \multirow[b]{2}{*}{ hi } & \multicolumn{4}{|c|}{ Estatísticas de acurácia } & \multicolumn{5}{|c|}{ Ranking } \\
\hline & $\mathrm{RP}$ & $\mathrm{D}$ & SSRR & $\mathrm{DP}$ & $\mathrm{RP}$ & $\mathrm{D}$ & SSRR & DP & Nota ranking. $h_{i}^{-1}$ \\
\hline 0,00 & 0,0000 & 0,0000 & 0,0000 & 0,0000 & 1,0 & 1,0 & 1,0 & 1,0 & 4,0 \\
\hline 0,25 & 4,2694 & 0,0001 & 2,8344 & 0,0010 & 2,0 & 3,0 & 2,0 & 1,0 & 8,0 \\
\hline 0,50 & $-0,2254$ & $-0,0004$ & 4,8058 & 0,0017 & 1,0 & 1,0 & 1,0 & 1,0 & 4,0 \\
\hline 0,75 & 1,4391 & $-0,0003$ & 2,1446 & 0,0023 & 1,0 & 1,0 & 2,0 & 1,0 & 5,0 \\
\hline 1,00 & $-0,8476$ & $-0,0008$ & 3,8916 & 0,0027 & 2,0 & 1,0 & 1,0 & 1,0 & 5,0 \\
\hline 1,30 & $-0,5880$ & $-0,0010$ & 3,4300 & 0,0030 & 4,0 & 1,0 & 1,0 & 1,0 & 7,0 \\
\hline 1,50 & 0,5862 & $-0,0008$ & 1,6742 & 0,0033 & 1,0 & 1,0 & 1,0 & 2,0 & 5,0 \\
\hline 2,00 & 0,4796 & $-0,0012$ & 2,8433 & 0,0037 & 2,0 & 1,0 & 1,0 & 1,0 & 5,0 \\
\hline 2,50 & 0,8206 & $-0,0013$ & 1,4362 & 0,0044 & 2,0 & 1,0 & 1,0 & 1,0 & 5,0 \\
\hline 3,00 & 1,6966 & $-0,0012$ & 2,5957 & 0,0046 & 2,0 & 1,0 & 1,0 & 1,0 & 5,0 \\
\hline 3,50 & 0,8899 & $-0,0016$ & 1,4116 & 0,0056 & 2,0 & 1,0 & 2,0 & 1,0 & 6,0 \\
\hline 4,00 & 2,0553 & $-0,0013$ & 2,6381 & 0,0056 & 2,0 & 1,0 & 2,0 & 1,0 & 6,0 \\
\hline 4,50 & 0,5699 & $-0,0020$ & 1,5182 & 0,0070 & 2,0 & 1,0 & 2,0 & 2,0 & 7,0 \\
\hline 5,00 & 2,0752 & $-0,0016$ & 2,8864 & 0,0068 & 2,0 & 1,0 & 2,0 & 2,0 & 7,0 \\
\hline 5,50 & 0,0060 & $-0,0026$ & 1,6303 & 0,0085 & 2,0 & 1,0 & 2,0 & 1,0 & 6,0 \\
\hline 6,00 & $-0,0891$ & $-0,0025$ & 2,3508 & 0,0082 & 2,0 & 1,0 & 1,0 & 1,0 & 5,0 \\
\hline 6,50 & $-1,9860$ & $-0,0036$ & 1,5172 & 0,0101 & 2,0 & 1,0 & 1,0 & 1,0 & 5,0 \\
\hline 7,00 & $-1,5900$ & $-0,0034$ & 2,2445 & 0,0097 & 2,0 & 1,0 & 1,0 & 1,0 & 5,0 \\
\hline 7,50 & $-3,9320$ & $-0,0052$ & 1,5053 & 0,0120 & 2,0 & 1,0 & 1,0 & 1,0 & 5,0 \\
\hline 8,00 & $-3,0530$ & $-0,0046$ & 2,1901 & 0,0112 & 2,0 & 1,0 & 1,0 & 1,0 & 5,0 \\
\hline 8,50 & $-6,3380$ & $-0,0072$ & 1,4118 & 0,0135 & 2,0 & 1,0 & 1,0 & 1,0 & 5,0 \\
\hline 9,00 & $-6,0500$ & $-0,0068$ & 1,7238 & 0,0123 & 2,0 & 1,0 & 1,0 & 1,0 & 5,0 \\
\hline 9,50 & $-9,3370$ & $-0,0102$ & 1,2418 & 0,0147 & 2,0 & 1,0 & 1,0 & 1,0 & 5,0 \\
\hline 10,00 & $-7,8280$ & $-0,0086$ & 1,6382 & 0,0132 & 2,0 & 1,0 & 1,0 & 1,0 & 5,0 \\
\hline 10,50 & $-10,8300$ & $-0,0123$ & 1,2470 & 0,0160 & 2,0 & 1,0 & 1,0 & 1,0 & 5,0 \\
\hline 11,00 & $-8,0260$ & $-0,0095$ & 1,3130 & 0,0141 & 2,0 & 1,0 & 1,0 & 1,0 & 5,0 \\
\hline 11,50 & $-12,0700$ & $-0,0152$ & 0,9618 & 0,0180 & 2,0 & 1,0 & 1,0 & 1,0 & 5,0 \\
\hline 12,00 & $-9,7600$ & $-0,0119$ & 1,1850 & 0,0158 & 2,0 & 1,0 & 1,0 & 1,0 & 5,0 \\
\hline 12,50 & $-14,7500$ & $-0,0191$ & 0,9266 & 0,0192 & 2,0 & 1,0 & 1,0 & 1,0 & 5,0 \\
\hline 13,00 & $-15,4900$ & $-0,0197$ & 1,1384 & 0,0186 & 2,0 & 1,0 & 1,0 & 1,0 & 5,0 \\
\hline 13,50 & $-12,1200$ & $-0,0182$ & 0,1600 & 0,0155 & 2,0 & 1,0 & 1,0 & 1,0 & 5,0 \\
\hline 14,00 & $-15,2200$ & $-0,0198$ & 0,3325 & 0,0157 & 2,0 & 1,0 & 1,0 & 1,0 & 5,0 \\
\hline 14,50 & $-18,8300$ & $-0,0336$ & 0,0355 & . & 2,0 & 1,0 & 1,0 & 0,0 & 4,0 \\
\hline 15,00 & $-17,9700$ & $-0,0264$ & 0,0648 & 0,0105 & 2,0 & 1,0 & 1,0 & 2,0 & 6,0 \\
\hline 15,50 & $-18,8300$ & $-0,0339$ & 0,0355 & . & 2,0 & 1,0 & 1,0 & 0,0 & 4,0 \\
\hline 16,00 & $-18,8400$ & $-0,0340$ & 0,0355 & . & 2,0 & 1,0 & 1,0 & 0,0 & 4,0 \\
\hline Total & & & & & & & & & 188,0 \\
\hline
\end{tabular}

Em que: $h_{i}, R P, D, S S R R$ e $D P$ foram definidos anteriormente. 
ACTA

AMAZONICA

Tabela 10 - Ranking da acurácia para o modelo Hradetzky (1976) para os volumes estimados ao longo do fuste das árvores de Tectona grandis L.f., para o conjunto de dados dos quatro povoamentos estudados.

\begin{tabular}{|c|c|c|c|c|c|c|c|c|c|}
\hline \multirow[b]{2}{*}{ hi } & \multicolumn{4}{|c|}{ Estatísticas de acurácia } & \multicolumn{5}{|c|}{ Ranking } \\
\hline & $\mathrm{RP}$ & $\mathrm{D}$ & SSRR & $\mathrm{DP}$ & $\mathrm{RP}$ & $\mathrm{D}$ & SSRR & $\mathrm{DP}$ & Nota ranking. $h_{i}^{-1}$ \\
\hline 0,00 & 0,0000 & 0,0000 & 0,0000 & 0,0000 & 1,0 & 1,0 & 1,0 & 1,0 & 4,0 \\
\hline 0,25 & 5,2944 & 0,0001 & 2,9312 & 0,0010 & 1,0 & 1,0 & 1,0 & 2,0 & 5,0 \\
\hline 0,50 & 2,0658 & $-0,0002$ & 4,7712 & 0,0016 & 3,0 & 4,0 & 2,0 & 2,0 & 11,0 \\
\hline 0,75 & 4,0754 & 0,0000 & 2,1730 & 0,0022 & 1,0 & 4,0 & 1,0 & 3,0 & 9,0 \\
\hline 1,00 & 1,7603 & $-0,0004$ & 3,6443 & 0,0025 & 3,0 & 3,0 & 2,0 & 3,0 & 11,0 \\
\hline 1,30 & 1,6304 & $-0,0005$ & 3,1481 & 0,0028 & 3,0 & 2,0 & 2,0 & 3,0 & 10,0 \\
\hline 1,50 & 2,4929 & $-0,0003$ & 1,5653 & 0,0030 & 1,0 & 4,0 & 2,0 & 3,0 & 10,0 \\
\hline 2,00 & 1,6088 & $-0,0008$ & 2,6123 & 0,0034 & 2,0 & 2,0 & 2,0 & 2,0 & 8,0 \\
\hline 2,50 & 1,4163 & $-0,0010$ & 1,3569 & 0,0041 & 1,0 & 2,0 & 2,0 & 2,0 & 7,0 \\
\hline 3,00 & 1,9854 & $-0,0011$ & 2,5389 & 0,0044 & 1,0 & 2,0 & 2,0 & 2,0 & 7,0 \\
\hline 3,50 & 1,0631 & $-0,0015$ & 1,4143 & 0,0055 & 1,0 & 2,0 & 1,0 & 2,0 & 6,0 \\
\hline 4,00 & 2,2442 & $-0,0013$ & 2,6959 & 0,0056 & 1,0 & 1,0 & 1,0 & 1,0 & 4,0 \\
\hline 4,50 & 0,8165 & $-0,0019$ & 1,5488 & 0,0070 & 1,0 & 2,0 & 1,0 & 1,0 & 5,0 \\
\hline 5,00 & 2,3921 & $-0,0015$ & 2,9441 & 0,0068 & 1,0 & 2,0 & 1,0 & 1,0 & 5,0 \\
\hline 5,50 & 0,3984 & $-0,0024$ & 1,6360 & 0,0084 & 1,0 & 2,0 & 1,0 & 2,0 & 6,0 \\
\hline 6,00 & 0,3504 & $-0,0023$ & 2,3413 & 0,0082 & 2,0 & 2,0 & 2,0 & 2,0 & 8,0 \\
\hline 6,50 & $-1,5140$ & $-0,0033$ & 1,4829 & 0,0100 & 2,0 & 2,0 & 2,0 & 2,0 & 8,0 \\
\hline 7,00 & $-1,1140$ & $-0,0031$ & 2,1837 & 0,0095 & 2,0 & 2,0 & 2,0 & 2,0 & 8,0 \\
\hline 7,50 & $-3,4530$ & $-0,0048$ & 1,4537 & 0,0118 & 2,0 & 2,0 & 2,0 & 2,0 & 8,0 \\
\hline 8,00 & $-2,5960$ & $-0,0043$ & 2,1194 & 0,0110 & 2,0 & 2,0 & 2,0 & 2,0 & 8,0 \\
\hline 8,50 & $-5,9050$ & $-0,0069$ & 1,3610 & 0,0133 & 2,0 & 2,0 & 2,0 & 2,0 & 8,0 \\
\hline 9,00 & $-5,6360$ & $-0,0064$ & 1,6602 & 0,0121 & 2,0 & 2,0 & 2,0 & 2,0 & 8,0 \\
\hline 9,50 & $-8,9560$ & $-0,0098$ & 1,1986 & 0,0145 & 2,0 & 2,0 & 2,0 & 2,0 & 8,0 \\
\hline 10,00 & $-7,4860$ & $-0,0083$ & 1,5865 & 0,0131 & 2,0 & 2,0 & 2,0 & 2,0 & 8,0 \\
\hline 10,50 & $-10,4900$ & $-0,0120$ & 1,2117 & 0,0159 & 2,0 & 2,0 & 2,0 & 2,0 & 8,0 \\
\hline 11,00 & $-7,7210$ & $-0,0092$ & 1,2778 & 0,0140 & 2,0 & 2,0 & 2,0 & 2,0 & 8,0 \\
\hline 11,50 & $-11,7600$ & $-0,0149$ & 0,9387 & 0,0179 & 2,0 & 2,0 & 2,0 & 2,0 & 8,0 \\
\hline 12,00 & $-9,4310$ & $-0,0115$ & 1,1536 & 0,0158 & 2,0 & 2,0 & 2,0 & 2,0 & 8,0 \\
\hline 12,50 & $-14,4000$ & $-0,0187$ & 0,9016 & 0,0191 & 2,0 & 2,0 & 2,0 & 2,0 & 8,0 \\
\hline 13,00 & $-15,1100$ & $-0,0192$ & 1,1044 & 0,0185 & 2,0 & 2,0 & 2,0 & 2,0 & 8,0 \\
\hline 13,50 & $-11,7800$ & $-0,0177$ & 0,1543 & 0,0154 & 2,0 & 2,0 & 2,0 & 2,0 & 8,0 \\
\hline 14,00 & $-14,8100$ & $-0,0193$ & 0,3208 & 0,0156 & 2,0 & 2,0 & 2,0 & 2,0 & 8,0 \\
\hline 14,50 & $-18,4400$ & $-0,0329$ & 0,0340 & . & 2,0 & 2,0 & 2,0 & 0,0 & 6,0 \\
\hline 15,00 & $-17,5300$ & $-0,0257$ & 0,0616 & 0,0102 & 2,0 & 2,0 & 2,0 & 3,0 & 9,0 \\
\hline 15,50 & $-18,3500$ & $-0,0330$ & 0,0337 & . & 2,0 & 2,0 & 2,0 & 0,0 & 6,0 \\
\hline 16,00 & $-18,3600$ & $-0,0331$ & 0,0337 & . & 2,0 & 2,0 & 2,0 & 0,0 & 6,0 \\
\hline Total & & & & & & & & & 271,0 \\
\hline
\end{tabular}

Em que: $h_{i}, R P, D, S S R R$ e $D P$ foram definidos anteriormente. 
Tabela 11 - Ranking da acurácia para o modelo Goulding \& Murray (1976) aplicado com volume estimado, para os volumes estimados ao longo do fuste das árvores de Tectona grandis L.f., para o conjunto de dados dos quatro povoamentos estudados.

\begin{tabular}{|c|c|c|c|c|c|c|c|c|c|}
\hline \multirow[b]{2}{*}{ hi } & \multicolumn{4}{|c|}{ Estatísticas de acurácia } & \multicolumn{5}{|c|}{ Ranking } \\
\hline & $\mathrm{RP}$ & $\mathrm{D}$ & SSRR & $\mathrm{DP}$ & $\mathrm{RP}$ & $\mathrm{D}$ & SSRR & $\mathrm{DP}$ & Nota ranking. $h_{i}^{-1}$ \\
\hline 0,00 & 0,0000 & 0,0000 & 0,0000 & 0,0000 & 1,0 & 1,0 & 1,0 & 1,0 & 4,0 \\
\hline 0,25 & $-3,4120$ & $-0,0001$ & 2,4306 & 0,0008 & 3,0 & 2,0 & 3,0 & 4,0 & 12,0 \\
\hline 0,50 & $-5,3910$ & $-0,0003$ & 3,9756 & 0,0013 & 2,0 & 3,0 & 3,0 & 4,0 & 12,0 \\
\hline 0,75 & $-0,6227$ & 0,0002 & 1,5315 & 0,0019 & 3,0 & 3,0 & 3,0 & 4,0 & 13,0 \\
\hline 1,00 & $-3,3180$ & $-0,0003$ & 2,5579 & 0,0021 & 2,0 & 4,0 & 4,0 & 4,0 & 14,0 \\
\hline 1,30 & $-2,6250$ & $-0,0002$ & 2,0323 & 0,0024 & 2,0 & 4,0 & 4,0 & 4,0 & 14,0 \\
\hline 1,50 & $-0,1338$ & 0,0004 & 1,0517 & 0,0029 & 3,0 & 3,0 & 3,0 & 4,0 & 13,0 \\
\hline 2,00 & $-1,3800$ & $-0,0001$ & 1,2984 & 0,0025 & 3,0 & 4,0 & 4,0 & 4,0 & 15,0 \\
\hline 2,50 & 0,0421 & 0,0004 & 0,7150 & 0,0031 & 4,0 & 4,0 & 3,0 & 4,0 & 15,0 \\
\hline 3,00 & $-0,3557$ & 0,0001 & 0,9647 & 0,0028 & 4,0 & 3,0 & 3,0 & 4,0 & 14,0 \\
\hline 3,50 & $-0,0119$ & 0,0005 & 0,6480 & 0,0037 & 4,0 & 4,0 & 3,0 & 3,0 & 14,0 \\
\hline 4,00 & 0,0676 & 0,0003 & 0,9128 & 0,0034 & 4,0 & 3,0 & 3,0 & 3,0 & 13,0 \\
\hline 4,50 & $-0,1466$ & 0,0004 & 0,6554 & 0,0044 & 4,0 & 4,0 & 3,0 & 3,0 & 14,0 \\
\hline 5,00 & 0,3445 & 0,0003 & 0,9640 & 0,0039 & 4,0 & 3,0 & 3,0 & 3,0 & 13,0 \\
\hline 5,50 & 0,0300 & 0,0004 & 0,6855 & 0,0051 & 3,0 & 4,0 & 3,0 & 3,0 & 13,0 \\
\hline 6,00 & 0,5364 & 0,0005 & 0,8500 & 0,0047 & 1,0 & 3,0 & 3,0 & 3,0 & 10,0 \\
\hline 6,50 & $-0,4172$ & 0,0003 & 0,6046 & 0,0060 & 4,0 & 4,0 & 3,0 & 3,0 & 14,0 \\
\hline 7,00 & 0,5246 & 0,0006 & 0,8353 & 0,0055 & 3,0 & 3,0 & 3,0 & 3,0 & 12,0 \\
\hline 7,50 & $-0,4530$ & 0,0003 & 0,5646 & 0,0069 & 4,0 & 4,0 & 3,0 & 3,0 & 14,0 \\
\hline 8,00 & 0,5068 & 0,0007 & 0,8098 & 0,0063 & 3,0 & 3,0 & 3,0 & 3,0 & 12,0 \\
\hline 8,50 & $-0,9310$ & 0,0002 & 0,4895 & 0,0078 & 3,0 & 4,0 & 3,0 & 3,0 & 13,0 \\
\hline 9,00 & $-0,0515$ & 0,0006 & 0,5079 & 0,0071 & 4,0 & 4,0 & 3,0 & 3,0 & 14,0 \\
\hline 9,50 & $-0,9580$ & 0,0002 & 0,3480 & 0,0087 & 3,0 & 4,0 & 3,0 & 3,0 & 13,0 \\
\hline 10,00 & $-0,0370$ & 0,0007 & 0,4537 & 0,0078 & 4,0 & 3,0 & 3,0 & 3,0 & 13,0 \\
\hline 10,50 & $-0,7264$ & 0,0003 & 0,3159 & 0,0095 & 3,0 & 4,0 & 3,0 & 3,0 & 13,0 \\
\hline 11,00 & 0,9478 & 0,0014 & 0,3023 & 0,0078 & 3,0 & 3,0 & 3,0 & 3,0 & 12,0 \\
\hline 11,50 & 0,1335 & 0,0010 & 0,1668 & 0,0100 & 4,0 & 3,0 & 3,0 & 3,0 & 13,0 \\
\hline 12,00 & 0,9943 & 0,0016 & 0,2571 & 0,0090 & 3,0 & 3,0 & 3,0 & 3,0 & 12,0 \\
\hline 12,50 & $-0,3252$ & 0,0000 & 0,1244 & 0,0094 & 4,0 & 4,0 & 3,0 & 3,0 & 14,0 \\
\hline 13,00 & $-1,3790$ & $-0,0011$ & 0,1641 & 0,0098 & 3,0 & 3,0 & 3,0 & 3,0 & 12,0 \\
\hline 13,50 & 2,8861 & 0,0046 & 0,0238 & 0,0079 & 3,0 & 3,0 & 3,0 & 3,0 & 12,0 \\
\hline 14,00 & $-0,8559$ & $-0,0004$ & 0,0574 & 0,0104 & 3,0 & 3,0 & 3,0 & 3,0 & 12,0 \\
\hline 14,50 & 5,3566 & 0,0096 & 0,0029 & . & 3,0 & 3,0 & 3,0 & 0,0 & 9,0 \\
\hline 15,00 & $-0,1386$ & 0,0017 & 0,0061 & 0,0112 & 3,0 & 3,0 & 3,0 & 1,0 & 10,0 \\
\hline 15,50 & 5,3526 & 0,0096 & 0,0029 & . & 3,0 & 3,0 & 3,0 & 0,0 & 9,0 \\
\hline 16,00 & 5,3456 & 0,0097 & 0,0029 & . & 3,0 & 3,0 & 3,0 & 0,0 & 9,0 \\
\hline Total & & & & & & & & & 445,0 \\
\hline
\end{tabular}


Tabela 12 - Ranking da acurácia para o modelo Goulding \& Murray (1976) aplicado com volume real, para os volumes estimados ao longo do fuste das árvores de Tectona grandis L.f., para 0 conjunto de dados dos quatro povoamentos estudados.

\begin{tabular}{|c|c|c|c|c|c|c|c|c|c|}
\hline \multirow[b]{2}{*}{ hi } & \multicolumn{4}{|c|}{ Estatísticas de acurácia } & \multicolumn{5}{|c|}{ Ranking } \\
\hline & $\mathrm{RP}$ & $\mathrm{D}$ & SSRR & $\mathrm{DP}$ & $\mathrm{RP}$ & $\mathrm{D}$ & SSRR & $\mathrm{DP}$ & Nota ranking. $h_{i}^{-1}$ \\
\hline 0,00 & 0,0000 & 0,0000 & 0,0000 & 0,0000 & 1,0 & 1,0 & 1,0 & 1,0 & 4,0 \\
\hline 0,25 & $-3,1650$ & 0,0000 & 2,0727 & 0,0008 & 4,0 & 4,0 & 4,0 & 3,0 & 15,0 \\
\hline 0,50 & $-6,4590$ & $-0,0004$ & 3,9069 & 0,0015 & 1,0 & 1,0 & 4,0 & 3,0 & 9,0 \\
\hline 0,75 & $-0,4792$ & 0,0002 & 1,3543 & 0,0023 & 4,0 & 2,0 & 4,0 & 2,0 & 12,0 \\
\hline 1,00 & $-4,4320$ & $-0,0004$ & 2,6066 & 0,0025 & 1,0 & 3,0 & 3,0 & 3,0 & 10,0 \\
\hline 1,30 & $-3,7680$ & $-0,0003$ & 2,1543 & 0,0029 & 1,0 & 3,0 & 3,0 & 2,0 & 9,0 \\
\hline 1,50 & $-0,0527$ & 0,0005 & 0,9724 & 0,0036 & 4,0 & 2,0 & 4,0 & 1,0 & 11,0 \\
\hline 2,00 & $-2,4990$ & $-0,0002$ & 1,3339 & 0,0031 & 1,0 & 3,0 & 3,0 & 3,0 & 10,0 \\
\hline 2,50 & 0,2032 & 0,0005 & 0,5143 & 0,0035 & 3,0 & 3,0 & 4,0 & 3,0 & 13,0 \\
\hline 3,00 & $-1,3930$ & 0,0000 & 0,7350 & 0,0029 & 3,0 & 4,0 & 4,0 & 3,0 & 14,0 \\
\hline 3,50 & 0,2361 & 0,0006 & 0,3000 & 0,0031 & 3,0 & 3,0 & 4,0 & 4,0 & 14,0 \\
\hline 4,00 & $-0,8924$ & 0,0001 & 0,4309 & 0,0026 & 3,0 & 4,0 & 4,0 & 4,0 & 15,0 \\
\hline 4,50 & 0,1715 & 0,0006 & 0,1801 & 0,0027 & 3,0 & 3,0 & 4,0 & 4,0 & 14,0 \\
\hline 5,00 & $-0,5435$ & 0,0002 & 0,2492 & 0,0024 & 3,0 & 4,0 & 4,0 & 4,0 & 15,0 \\
\hline 5,50 & 0,2814 & 0,0006 & 0,1020 & 0,0024 & 2,0 & 3,0 & 4,0 & 4,0 & 13,0 \\
\hline 6,00 & $-0,0267$ & 0,0003 & 0,1355 & 0,0022 & 4,0 & 4,0 & 4,0 & 4,0 & 16,0 \\
\hline 6,50 & 0,4862 & 0,0006 & 0,0560 & 0,0022 & 3,0 & 3,0 & 4,0 & 4,0 & 14,0 \\
\hline 7,00 & 0,1975 & 0,0004 & 0,0721 & 0,0020 & 4,0 & 4,0 & 4,0 & 4,0 & 16,0 \\
\hline 7,50 & 0,4612 & 0,0006 & 0,0280 & 0,0019 & 3,0 & 3,0 & 4,0 & 4,0 & 14,0 \\
\hline 8,00 & 0,3665 & 0,0005 & 0,0375 & 0,0017 & 4,0 & 4,0 & 4,0 & 4,0 & 16,0 \\
\hline 8,50 & 0,5002 & 0,0006 & 0,0148 & 0,0016 & 4,0 & 3,0 & 4,0 & 4,0 & 15,0 \\
\hline 9,00 & 0,5740 & 0,0006 & 0,0217 & 0,0015 & 3,0 & 3,0 & 4,0 & 4,0 & 14,0 \\
\hline 9,50 & 0,5565 & 0,0007 & 0,0082 & 0,0014 & 4,0 & 3,0 & 4,0 & 4,0 & 15,0 \\
\hline 10,00 & 0,5739 & 0,0006 & 0,0121 & 0,0012 & 3,0 & 4,0 & 4,0 & 4,0 & 15,0 \\
\hline 10,50 & 0,4673 & 0,0006 & 0,0042 & 0,0011 & 4,0 & 3,0 & 4,0 & 4,0 & 15,0 \\
\hline 11,00 & 0,4284 & 0,0005 & 0,0058 & 0,0010 & 4,0 & 4,0 & 4,0 & 4,0 & 16,0 \\
\hline 11,50 & 0,4244 & 0,0006 & 0,0021 & 0,0010 & 3,0 & 4,0 & 4,0 & 4,0 & 15,0 \\
\hline 12,00 & 0,3652 & 0,0005 & 0,0024 & 0,0008 & 4,0 & 4,0 & 4,0 & 4,0 & 16,0 \\
\hline 12,50 & 0,3902 & 0,0005 & 0,0009 & 0,0008 & 3,0 & 3,0 & 4,0 & 4,0 & 14,0 \\
\hline 13,00 & 0,3345 & 0,0004 & 0,0008 & 0,0006 & 4,0 & 4,0 & 4,0 & 4,0 & 16,0 \\
\hline 13,50 & 0,5061 & 0,0007 & 0,0003 & 0,0007 & 4,0 & 4,0 & 4,0 & 4,0 & 16,0 \\
\hline 14,00 & 0,2221 & 0,0003 & 0,0001 & 0,0004 & 4,0 & 4,0 & 4,0 & 4,0 & 16,0 \\
\hline 14,50 & 0,0020 & 0,0000 & 0,0000 & . & 4,0 & 4,0 & 4,0 & 0,0 & 12,0 \\
\hline 15,00 & 0,0272 & 0,0000 & 0,0000 & 0,0000 & 4,0 & 4,0 & 4,0 & 4,0 & 16,0 \\
\hline 15,50 & $-0,0022$ & 0,0000 & 0,0000 & . & 4,0 & 4,0 & 4,0 & 0,0 & 12,0 \\
\hline 16,00 & $-0,0097$ & 0,0000 & 0,0000 & . & 4,0 & 4,0 & 4,0 & 0,0 & 12,0 \\
\hline Total & & & & & & & & & 489,0 \\
\hline
\end{tabular}

Em que: $h$, RP, D, SSRR e DP foram definidos anteriormente. 
foram com valores 3,0 e 4,0. O modelo gerou excelentes estimativas em qualquer porção do fuste, sendo, portanto, a equação mais estável. Na maioria das posiçôes, os valores de desvio (D) foram próximos de zero e as demais estatísticas apresentaram excelentes resultados.

As estatísticas para o caso fictício, em que para o modelo Goulding \& Murray (1976), foi considerado o volume real, os resultados de desvio (D) foram zero em diversas posiçōes e, nas demais, próximo de zero.

Observou-se que o modelo Goulding \& Murray (1976) apresentou o melhor desempenho em $100 \%$ das posiçōes avaliadas. Considerando a base de dados estudada, este modelo revelou um desempenho significativamente superior aos demais modelos testados, para o estudo em questão.

Caso fossem comparados apenas os modelos Schöepfer (1966) e Hradetzky (1976), as estimativas geradas por Hradetzky (1976), seriam predominantemente melhores nas porçôes superiores e inferiores do fuste. Na porção mediana, entre as alturas de quatro a seis metros, o modelo Schöepfer (1966) apresentaria melhor desempenho. Estes resultados indicam fragilidade do modelo Schöepfer (1966) em estimar os valores de volume para as porções extremas do fuste. Para o modelo Hradetzky (1976), um artifício do procedimento estatístico Stepwise poderia ser empregado para melhorar a performance das estimativas volumétricas na porção mediana do fuste, que seria a fixação de variáveis com potências representativas para determinada faixa de altura ao longo do fuste.

$\mathrm{O}$ ranking para as estimativas de diâmetros e volumes nas várias porçôes do fuste indicou a superioridade do modelo Goulding \& Murray (1976). Provavelmente, um dos motivos que levou ao bom desempenho do modelo foi o vínculo estabelecido com os volumes estimados, pois os bons resultados das estimativas de volume alcançado pelo modelo volumétrico selecionado certamente contribuíram para os resultados da função de afilamento de Goulding \& Murray (1976).

$\mathrm{Na}$ Figura 2 estão os gráficos de perfis para as estimativas de volume ao longo do fuste, considerando os três modelos avaliados e para o modelo Goulding \& Murray (1976) com volume real.

Os modelos Schöepfer (1976) e Hradetzky (1976) apresentaram menor aderência com as estimativas do volume real, sendo que, a partir da altura absoluta de cinco metros, as estimativas sempre foram superestimadas e, nas alturas inferiores a cinco metros, ora os valores foram subestimados, ora superestimados.

O perfil apresentado pelo modelo Goulding \& Murray (1976) com volume estimado reforça os resultados das estatísticas de acurácia, que aponta ser a melhor função de afilamento. Em todas as porções ao longo do fuste sempre ocorreram valores de volumes superestimados ou subestimados. No entanto, a diferença entre os valores do volume real e estimado sempre foi pequena, sendo quase imperceptível no gráfico do perfil da árvore média. Nas últimas alturas, o modelo gerou maiores subestimativas, as quais são mais evidenciadas nas alturas de 13,5, 14 e 15,5 metros.

Já para o perfil da árvore média gerada pelo modelo Goulding \& Murray (1976) com volume real, as estimativas foram também ora subestimadas, ora superestimadas. Porém, ao se observar o gráfico do perfil, percebe-se que o volume estimado pela função de afilamento praticamente recobre a linha do perfil da árvore média gerada pelos volumes reais. As diferenças na ordem de
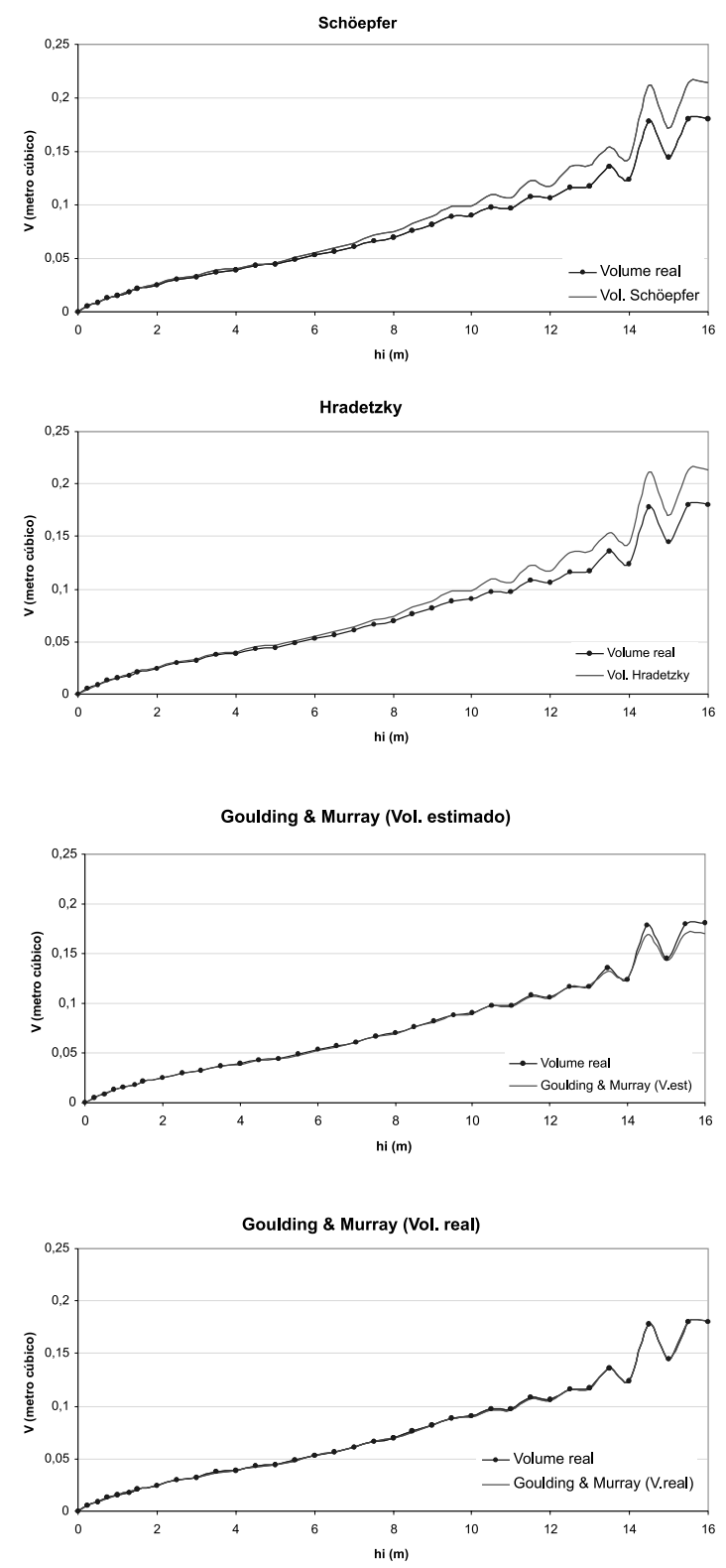

Figura 2 - Volumes médios reais e estimados pelos modelos polinomiais de Schöepfer (1966), Hradetzky (1976) e Goulding \& Murray (1976), para 0 conjunto de dados dos quatro povoamentos de Tectona grandis L.f. 
fração de milhar para os volumes estimados tornam imperceptível a oscilação da linha de volume estimado (vermelho) e volume real (azul).

\section{TESTE DE IDENTIDADE DE MODELOS.}

As onze combinaçôes apresentadas na Tabela 13 representam todos os possíveis agrupamentos para a formação de modelos reduzidos. Também são apresentados os valores de variância da regressão, que possibilitou o ajuste dos polinômios e os respectivos valores de $\mathrm{F}_{\text {calculado }}$ e $\mathrm{F}_{\text {tabelado }}$ para uma significância de 5\%. Dessa maneira, sempre que o valor de $\mathrm{F}_{\text {calculado }}$ for maior que o valor de $\mathrm{F}_{\text {tabelado }}$, considera-se o teste significativo; portanto, a 5\% de significância aceita-se a hipótese $\mathrm{H}_{1}$, ou seja, a soma dos quadrados dos resíduos para cada um dos povoamentos estudados (modelo completo - U) difere estatisticamente da soma dos quadrados dos resíduos para o modelo reduzido $(w)$. Da mesma forma, quando o valor de $\mathrm{F}_{\text {calculado }}$ for menor que o valor de $\mathrm{F}_{\text {tabelado }}$ o teste é considerado não significativo, ou seja, aceita-se $\mathrm{H}_{0}$, o que significa que, não existe diferença significativa entre os modelos completo $(\mathrm{U})$ e reduzido $(w)$ na combinação avaliada.

Em todas as combinações testadas, somente a combinação das áreas 2 e 3 foi possível de agrupar. Percebe-se que as combinações que envolvem as áreas $\{1$ e 3$\}$ geraram altos valores para o $\mathrm{F}_{\text {calculado }}$, demonstrando que existe uma forte diferença entre estas duas áreas no que se refere ao afilamento das árvores como retratados pelo modelo de Goulding \& Murray (1976).

Os altos valores para $\mathrm{F}_{\text {calculado }}$ também são observados nas combinações das áreas $\{1$ e 4$\},\{1$ e 2$\},\{1,2,3$ e 4$\},\{1,2$ e 3$\},\{1$, 2 e 4$\}$ e $\{1,3$ e 4$\}$.
Os modelos que possibilitam os melhores ajustes geralmente possibilitam um menor número de combinações, demonstrando sua maior exigência em agrupar dados advindos de povoamentos diferentes.

Os parâmetros estimados e as estatísticas de precisão dos modelos ajustados individualmente para as áreas 1, 2, 3 e 4 e para a combinação $\{2$ e 3$\}$, a qual foi indicada pelo teste de identidade de modelo, encontram-se na Tabela 14.

\section{CONCLUSÕES}

O modelo polinomial Goulding \& Murray (1976) foi preciso e exato na estimativa dos diâmetros e volumes ao longo do fuste;

O volume real advindo da cubagem rigorosa é impraticável de ser utilizado no inventário florestal estabelecendo uma situação fictícia de uso e, portanto, não deve ser utilizado associado ao modelo de Goulding \& Murray (1976) nos testes de acurácia;

O polinômio de potências inteiras e fracionárias (Hradetzky, 1976) apresentou bons para retratar as extremidades inferiores $e$ superiores das árvores e resultados regulares para a porção intermediária;

O modelo polinomial Schöepfer (1966) apresentou dificuldade de modelar as extremidades das árvores propiciando as piores estimativas de diâmetro e volume;

O teste de identidade de modelo indicou que as árvores das áreas 2 e 3 apresentam a mesma tendência de forma ou afilamento. $\mathrm{E}$ a árvores das áreas 1 e 4 apresentam tendências diferentes em seu afilamento o que implica em ajustar modelos independentes para as áreas 1 e 4 e um único para o subgrupo 2 e 3, quando utilizado o modelo Goulding \& Murray (1976).

Tabela 13 - Resultado do teste de identidade de modelos por meio do teste F, para as diferentes combinações das quatro áreas estudadas. 0 modelo de afilamento testado foi Goulding \& Murray (1976)

\begin{tabular}{|c|c|c|c|c|c|}
\hline Combinações & $\mathbf{Q} \mathbf{M}_{\text {(diterença) }}$ & $\mathrm{QM}_{\text {(residuo) }}$ & $\mathrm{F}_{\text {(calculado) }}$ & F $5 \%_{\text {(tabelado) }}$ & Teste \\
\hline $1,2,3$ e 4 & 0,6118500 & 0,0728492 & 8,399 & 1,6073 & $S$ \\
\hline 1,2 e 3 & 0,7327750 & 0,0796150 & 9,204 & 1,7565 & $S$ \\
\hline 1,2 e 4 & 0,5552750 & 0,0795567 & 6,980 & 1,7566 & $S$ \\
\hline 1,3 e 4 & 0,7650000 & 0,0667089 & 11,468 & 1,7556 & S \\
\hline 2,3 e 4 & 0,2218583 & 0,0654157 & 3,392 & 1,7580 & S \\
\hline 1 e 2 & 0,4735500 & 0,0934381 & 5,068 & 2,1048 & S \\
\hline 1 e 3 & 1,1555000 & 0,0717718 & 16,100 & 2,1029 & S \\
\hline 1 e 4 & 0,7133333 & 0,0714337 & 9,986 & 2,1030 & $S$ \\
\hline 2 e 3 & 0,1443833 & 0,0759753 & 1,900 & 2,1084 & NS \\
\hline 2 e 4 & 0,2778833 & 0,0755200 & 3,680 & 2,1092 & $S$ \\
\hline 3 e 4 & 0,2326667 & 0,0533532 & 4,361 & 2,1045 & $S$ \\
\hline
\end{tabular}

Em que: $\mathrm{S}=$ significativo a 95\% de probabilidade pela distribuição $\mathrm{Fe} N \mathrm{NS}=$ não significativo a 95\% de probabilidade pela distribuição $\mathrm{F}$. 
Tabela 14 - Parâmetros estimados/calculados, potências e medidas de precisão para os modelos polinomiais de Goulding \& Murray (1976), para as áreas 1, 2, 3 e 4 individualmente e 0 conjunto das áreas 2 e 3, conforme indicação de agrupamento do teste de identidade de modelos

\begin{tabular}{|c|c|c|c|c|c|c|}
\hline Modelo & & râmetros & Potências & & $\mathbf{R}^{2}(\%)$ & $S_{y x}(\%)$ \\
\hline \multirow{7}{*}{ Área 1} & $\beta 1$ & 0,997878 & $p 1$ & 1 & & \\
\hline & $\beta 2$ & 1,182711 & $p 2$ & 2 & & \\
\hline & $\beta 3$ & $-3,30703$ & p3 & 11 & & \\
\hline & $\beta 4$ & 5,898165 & $p 4$ & 14 & $96,25 \%$ & $9,70 \%$ \\
\hline & $\beta 5$ & $-274,616$ & $p 5$ & 85 & & \\
\hline & $\beta 6$ & 572,2235 & $p 6$ & 90 & & \\
\hline & $\beta 7$ & $-298,153$ & $p 7$ & 95 & & \\
\hline \multirow{7}{*}{ Área 2} & $\beta 1$ & 1,378141 & $p 1$ & 1 & & \\
\hline & $\beta 2$ & 0,63393 & $p 2$ & 2 & & \\
\hline & $\beta 3$ & $-0,949554$ & $p 3$ & 11 & & \\
\hline & $\beta 4$ & 1,773435 & $p 4$ & 14 & $89,46 \%$ & $11,30 \%$ \\
\hline & $\beta 5$ & 866,5618 & $p 5$ & 85 & & \\
\hline & $\beta 6$ & $-1748,2283$ & $p 6$ & 90 & & \\
\hline & $\beta 7$ & 882,76992 & $p 7$ & 95 & & \\
\hline \multirow{7}{*}{ Área 3} & $\beta 1$ & 1,068054 & $p 1$ & 1 & & \\
\hline & $\beta 2$ & 1,237536 & $p 2$ & 2 & & \\
\hline & $\beta 3$ & $-3,439608$ & $p 3$ & 11 & & \\
\hline & $\beta 4$ & 5,095485 & $p 4$ & 14 & $97,31 \%$ & $7,16 \%$ \\
\hline & $\beta 5$ & $-51,615824$ & $p 5$ & 85 & & \\
\hline & $\beta 6$ & 107,96422 & $p 6$ & 90 & & \\
\hline & $\beta 7$ & $-56,24064$ & $p 7$ & 95 & & \\
\hline \multirow{7}{*}{ Área 4} & $\beta 1$ & 1,209732 & $p 1$ & 1 & & \\
\hline & $\beta 2$ & 0,900612 & $p 2$ & 2 & & \\
\hline & $\beta 3$ & $-1,748184$ & p3 & 11 & & \\
\hline & $\beta 4$ & 3,35343 & $p 4$ & 14 & $97,64 \%$ & $7,02 \%$ \\
\hline & $\beta 5$ & $-119,15902$ & $p 5$ & 85 & & \\
\hline & $\beta 6$ & 272,04268 & $p 6$ & 90 & & \\
\hline & $\beta 7$ & $-152,33856$ & $p 7$ & 95 & & \\
\hline \multirow{7}{*}{ Área 2 e 3} & $\beta 1$ & 1,11112 & $p 1$ & 1 & & \\
\hline & $\beta 2$ & 1,177716 & $p 2$ & 2 & & \\
\hline & $\beta 3$ & $-3,828936$ & p3 & 11 & & \\
\hline & $\beta 4$ & 5,58939 & $p 4$ & 14 & $96,87 \%$ & $7,73 \%$ \\
\hline & $\beta 5$ & $-52,740102$ & $p 5$ & 85 & & \\
\hline & $\beta 6$ & 108,91244 & $p 6$ & 90 & & \\
\hline & $\beta 7$ & $-56,185248$ & $p 7$ & 95 & & \\
\hline
\end{tabular}

Em que: $\beta_{\mathrm{s}}=$ parâmetros estimados, $\mathrm{p}_{\mathrm{is}}=$ potências selecionadas, $\mathrm{R}^{2}=$ coeficiente de determinação e $S_{\mathrm{yx}} \%=$ erro padrão residual percentual.

\section{BIBLIOGRAFIA CITADA}

Assis, A.L.; Scolforo, J.R.S.; Mello, J.M.; Acerbi Júnior, F.W.; Oliveira, A.D. 2001. Comparação de modelos polinomiais segmentados e não-segmentados na estimativa de diâmetros e volumes ao longo do fuste de Pinus taeda. Cerne, 7(1): 20-40.

Ferreira, M.Z. 2004. Estudo de funçôes de afilamento para representar o perfil e o volume do fuste de Pinus taeda $L$. Dissertação de Mestrado, Universidade Federal de Lavras, Lavras, Minas Gerais. 200pp.

Figueiredo Filho, A.; Borges, B.E.; Hitch, K.L. 1996. Taper equations for Pinus taeda plantations in southern Brazil. Forest Ecology and Management, 83(1/2): 36-46.

Fischer, F. 1997. Eficiência dos modelos polinomiais e das razôes de volume na estimativa volumétrica dos sortimentos e do perfil do fuste de Pinus taeda. Dissertação de Mestrado, Universidade Federal de Lavras, Lavras, Minas Gerais. 167 pp.

Goulding, C.J.; Murray, J.C. 1976. Polynomial taper equations that are compatible with tree volume equations. New Zealand Journal of Forest Science, 5(3): 313-322.

Graybill, J. 1976. Theory and application of the linear model. Belmont: Duxbury. Não paginado.

Hradetzky, J. 1976. Analyse und interpretation statistisher abränger keiten. (Biometrische Beiträge zu aktuellen forschungs projekten). Baden: Württemberg Mitteilungen der FVA. 146pp.

Husch, B.; Miller, C.I.; Beer, T.W. 1982. Forest mensuration. 3.ed.: J. Wiley \& Sons, New York. 402pp.

Parresol, B.R.; Hotvedt, J.E.; Cao, Q.V.A. 1987. Volume and taper prediction system for bald cypress. Canadian Journal of Forest Research, 17(3) :250-259.

Sas Institute. 1990. SAS User's guide: statistics. Release 6.03. Cary. 584pp.

Schöepfer,W. 1966. Automatisierung des Massen, Sorten und Wertberechnung stenender Waldbestande Schriftenreihe Bad. [S.I.]: Wurtt-Forstl., Não paginado.

Scolforo, J.R.S. 1997. Biometria florestal 2: técnicas de regressão aplicada para estimar: volume, biomassa, relação hipsométrica e múltiplos produtos de madeira. UFLA/FAEPE, Lavras. 292pp.

Scolforo, J.R.S.; Rios, M.S.; Oliveira, A.D. de; Mello, J.M. de; Maestri, R. 1998. Acuracidade de equaçóes de afilamento para representar o perfil do fuste de Pinus elliotti. Cerne, 4(1): 100122.

Scolforo, J.R.S. 2005. Biometria florestal: Parte I: modelos de regressão linear e não-linear; Parte II: modelos para relação hipsométrica, volume, afilamento, e peso de matéria seca. Lavras: UFLA/FAEPE, 352pp.

Recebido em 17/10/2005

Aceito em 13/10/2006 